Article

\title{
Compact Polarimetric Response of Rape (Brassica napus L.) at C-Band: Analysis and Growth Parameters Inversion
}

\author{
Wangfei Zhang ${ }^{1,2}$, Zengyuan $\mathrm{Li}^{1}$, Erxue Chen ${ }^{1, *}$, Yahong Zhang ${ }^{2}$, Hao Yang ${ }^{3}$, Lei Zhao ${ }^{1}$ \\ and Yongjie $\mathrm{Ji}^{2}$ \\ 1 Institute of Forest Resource Information Techniques, Chinese Academy of Forestry, Beijing 100091, China \\ mewhff@163.com (W.Z.); zengyuan.li@caf.ac.cn (Z.L.); zhaoleiiam@126.com (L.Z.) \\ 2 College of Forestry, Southwest Forestry University, Kunming 650224, China; yuezhizhuh@163.com (Y.Z.); \\ jiyongjie@live.cn (Y.J.) \\ 3 Beijing Research Center for Information Technology in Agriculture, Beijing Academy of Agriculture and \\ Forestry Sciences, Beijing 100097, China; younghow@163.com \\ * Correspondence: chenerx@caf.ac.cn; Tel./Fax: +86-10-6288-9164
}

Academic Editors: Nicolas Baghdadi and Prasad S. Thenkabail

Received: 22 March 2017; Accepted: 6 June 2017; Published: 11 June 2017

\begin{abstract}
Growth parameters like biomass, leaf area index (LAI) and stem height play an import role for crop monitoring and yield prediction. Compact polarimetric (CP) SAR has shown great potential and similar performance to fully-polarimetric (FP) SAR in crop mapping and phenology retrieval, but its potential in growth parameters inversion has not been fully explored. In this paper, a time series of images of CP SAR was simulated from five FP SAR data gathered during the entire growth season of rape. CP response of 27 parameters, relying on Stokes parameters and their child parameters, decomposition parameters and backscattering coefficients, were extracted and investigated as a function of days after sowing (DAS) during the whole rape growth cycle to interpret their sensitivity to each growth parameter. Then, random forest (RF) was chosen as an automatic approach for the growth parameters inversion method, and its results were compared with traditional single-parameter regression models. Most of the $\mathrm{CP}$ parameters showed high sensitivity with growth parameters and great potential for growth parameters inversion. Among all of the regression models, the quadratic regression model showed the best performance for all of the growth parameters inversion, the best result for biomass inversion was the third component of the Stokes parameters $\left(g_{3}\right)$ with $R^{2}$ of 0.765 and RMSE of $73.20 \mathrm{~g} / \mathrm{m}^{2}$. For LAI and stem height was one of the Stokes child parameters, the circular polarization ratio $\left(U_{c}\right)$, with $\mathrm{R}^{2}$ of 0.857 and 0.923 and RMSE of 0.66 and $18.71 \mathrm{~cm}$, respectively. RF showed the highest accuracy and smallest RMSE for all of three growth parameters inversion; $\mathrm{R}^{2}$ for biomass, LAI and stem height were 0.93, 0.96 and 0.95, respectively; RMSE were $46.24 \mathrm{~g} / \mathrm{m}^{2}, 0.25$ and $13.5 \mathrm{~cm}$, respectively. However, there are also some CP parameters, which showed low sensitivity to growth parameters, that had high importance for RF inversion. The results confirmed the potential of $\mathrm{CP}$ data and the RF method in growth parameters inversion, but they also confirmed that it was difficult to give a physical interpretation for the RF inversion model.
\end{abstract}

Keywords: compact polarimetric (CP) SAR; rape; Radarsat-2; day after sowing (DAS); random forest; Stokes parameters; polarimetric decomposition; backscatter coefficients

\section{Introduction}

Rape (Brassica napus L.) is surely one of the important oil plants in the world because it not only accounts for the main part of annual edible oil, but also constitutes the most promising clean 
fuels through biodiesel made from its seeds [1]. Stem height is a useful indicator of crops biomass; the biomass of crop vegetation plays an important role in plant monitoring and yield prediction. The leaf area index (LAI) is an important cultivation physiology parameter for the measurement of the reasonableness of the crop community. These growth parameters reflect the photosynthetic potential of crops at a certain period, which directly affect the biological and economic yield [2]. For the importance of these growth parameters for yield estimation, the sensitivity of climate change and crop monitoring, as well as the destructive and expensive procedure for directly measuring, there is a significant interest in this information collected by remote sensing techniques [3]. Various studies have been carried out using remote sensing to estimate biomass, LAI for various crops, based on vegetation indices from optical sensors with regression models or the vegetation radiation transfer function $[4,5]$. However, designed for a fixed period, these methods are inadequate for dynamic analysis of the growth parameter and their changes accurately and in a timely manner, especially for precision agriculture. Synthetic aperture radar (SAR) with an appropriate wavelength and polarization mode is sensitive to plant structure with its penetration ability into the plant crown layer [6]. Meanwhile, the short revisit times and full polarization modes of SAR made it possible to monitor, map and analyze the crop's entire growth cycle. Then, the final users can in a timely manner and accurately know the current situation of the crop along its cultivation [3]. Fully-polarimetric (FP) SAR, which embraces a quantitative measurement of the relative phase and amplitudes of the backscattered power, as well as the different scattering mechanisms extracted through polarimetric decomposition methods, has been extensively studied for applications such as crop-type classification and mapping [6-8], crop phenology monitoring [3,9] and growth parameters inversion [10-12]. Backscattering coefficients [13], polarization target decomposition components [14,15] and Stokes parameters [16-18] from FP SAR data have been proven to be the most elegant and promising parameters for target physical information and scattering mechanism interpretation. Lopez- Sanchez et al. analyzed several FP observables extracted from Radarsat-2 at C-band and showed the sensitivity of these observables to the rice growth cycle [3]. Research results from Agriculture and Agri-Food Canada also showed the potential and effectiveness of SAR data applied in crops' classification, growth parameters inversion and soil moisture inversion [19]. The identification accuracy for crop types in the C-band was about $85 \%$ and in X-band about $95 \%$ [20]. It also showed that backscatter coefficients were useful for crop biomass and LAI inversion with a higher saturation level [20]. The results also confirmed the possibility and accuracy of crops' identification with short wavelength SAR data. Although FP SAR has good performance in crops' monitoring, its known limitation is the reduced swath produced by the doubled pulse repetition frequency, which is employed to scan all combinations of transmitted/received polarization. To avoid such a constraint, compact polarimetry $(\mathrm{CP})$, transmitting only one polarization and receiving two orthogonal polarizations, has been proposed. Differing from conventional dual-polarization SAR, CP SAR requires that the relative phase between the two receive polarizations be retained $[17,18,21]$. Although there is reduced information of $\mathrm{CP}$ from its $2 \times 2$ covariance matrix of the backscattered field compared with fully-polarimetric data, it has shown great potential and similar performance to FP data in crop mapping, classification, phenology retrieval and crop monitoring [3,21-23]. CP decomposition methods proposed recently also extended the application of $\mathrm{CP}$ data $[21,24]$. Charbonneau et al. compared the performance of CP observables with FP data on crops' classification at different growth stages and found that CP Stokes parameters $\left(g_{0}, g_{1}, g_{2}, g_{3}\right)$ produced better classification results than FP data at the early growth stage of crops. CP Stokes child observables and CP decomposition components showed similar classification accuracy with FP using the Cloude-Pottier method [21]. Lopez-Sanchez et al. first reported the performance of CP SAR in rice phenology retrieval, but only exploited four $\mathrm{CP}$ parameters $\left(\mathrm{m}, \delta, \frac{\chi}{\alpha}, U_{c}\right)$ with rice phenological changes [3]. Yang et al. extended the research by using $11 \mathrm{CP}$ parameters $\left(\sigma_{\mathrm{RH}}, \sigma_{\mathrm{RV}}, \sigma_{\mathrm{RL}}, \sigma_{\mathrm{RR}}, \mathrm{m}, \delta, \frac{\chi}{\alpha}, U_{c}, P_{D \alpha}, P_{S \alpha}, P_{V \alpha}\right)$ and identified the rice phenological stages [23]. $\mathrm{CP}$ data were reported for rape growth parameters (biomass) inversion, but only $\mathrm{CP}$ decomposition parameters were applied for the inversion of these parameters, and only one CP parameters was used for biomass inversion [25]. Moreover, these previous studies on the 
inversion of growth parameters were limited to linear regression empirical models. To the best of our knowledge, no previous research has employed any automatic approach (e.g., support vector machines (SVM), artificial neural networks (ANN), random forest (RF)) for crop growth parameters inversion with $\mathrm{CP}$ parameters [3,4].

The present study aims at fully exploring CP SAR response on rape and finds the potential of the automatic approach for rape biomass, stem height and LAI inversion. Among the above-mentioned automatic approaches, RF has been regarded as one of the most precise prediction methods, which can model complex interactions among input variables, and it is also relatively robust in regard to outliers. Hence, RF was chosen for the three growth parameters' inversion in this paper. For the poor physical interpretation of the RF method for growth parameters inversion, the detailed analysis of CP parameters changing with the growth parameter during the whole growth cycle was also described in this paper. This paper is organized as follows. Section 2 briefly introduces the test site, SAR data, ground data and numerical scales of rape. Then, the SAR processing and CP SAR data simulation are detailed in Section 3. Section 4 presents the CP parameters divided into four groups. Section 5 describes the evolution of all $\mathrm{CP}$ parameters and growth parameters as a function of day after sowing (DAS). Section 6 proposes and tests the potential of the RF algorithm for rape biomass, stem height and LAI inversion. Finally, the main conclusions of this research are summarized in Section 7.

\section{Test Sites, SAR Data and Ground Measurement Campaign}

The test site is a farmland named Shangkuli of $2800 \mathrm{hm}^{2}$ in City Hailar, Inner Mongolia, the northeast of China, where spring rape (Brassica napus L.) and wheat (Triticum aestivum and Hordeum vulgare) are cultivated annually from May-September, approximately. Figure 1 shows the location of test site $\left(120.76^{\circ}\right.$ to $120.89^{\circ} \mathrm{E} ; 50.28^{\circ}$ to $\left.50.39^{\circ} \mathrm{N}\right)$. The Pauli basis RGB composite image in Figure 1 was obtained from a Radarsat- 2 image acquired on 23 May 2013. The specific variety of rape cultivated at the test site was called hybrid-5 rape, quite common in the north of China. Leached chernozem soil dominates this area, which has higher water retention. The terrain here is gentle with slopes less than $1 \%$. Climate in the test site is the cold temperate continental monsoon climate. It has a cold and dry long winter and warm and wet short summer, which supports one harvest per year. As the farming practice in this specific location, sowing was carried out by the planter XIAN-2BF-24C, which sows $0.425 \mathrm{~kg} / \mathrm{mu}$ over the fields. The cultivation campaign lasted about 115-140 days.

A set of five FP Radarsat-2 images was acquired over this area during the whole rape growing season, all of them with the same mode, beam and orbit pass, in order to build a time series dataset in the most consistent way. All of these images were provided in single look complex (SLC) format with a pixel size of $4.73 \mathrm{~m}$ and $4.96 \mathrm{~m}$ in the azimuth and slant range direction, respectively. Table 1 shows the image acquisition parameter details.

Table 1. The details of 5 Radarsat- 2 images.

\begin{tabular}{cc}
\hline Parameters & Values \\
\hline Polarization & Quad \\
Frequency & $5.405 \mathrm{GHz}$ \\
Incidence angle & $37.4-38.8$ \\
Range pixel spacing & $4.96 \mathrm{~m}$ \\
Azimuth pixel spacing & $4.73 \mathrm{~m}$ \\
Orbit direction & Ascending \\
Beam mode & FQ18 \\
\hline
\end{tabular}

Since one of the objectives of this study is to describe the dynamic change of $\mathrm{CP}$ parameters with rape growth parameter changes, it is better to describe the rape growth period with crop temporal evolution, which is usually described by a simple linear interpolator known as the general $\mathrm{BBCH}$ scale (from Biologische Bundesanstalt Bundessortenamt and CHemische Industrie) for cereals [26]. In this paper, 
the whole rape growth cycle was divided into $10 \mathrm{BBCH}$ stages according to the particular features and aspects of rape development as a function of time. However, because of the long revisit time of Radarsat-2 (24 days) acquired for this study, several BBCH stages were combined into a principal scale as (1) and (2) into P2, (3), (5), (6) into P3, and so on. The principal stages, the corresponding BBCH scales and the related acquired SAR images are introduced in detail in Table 2.

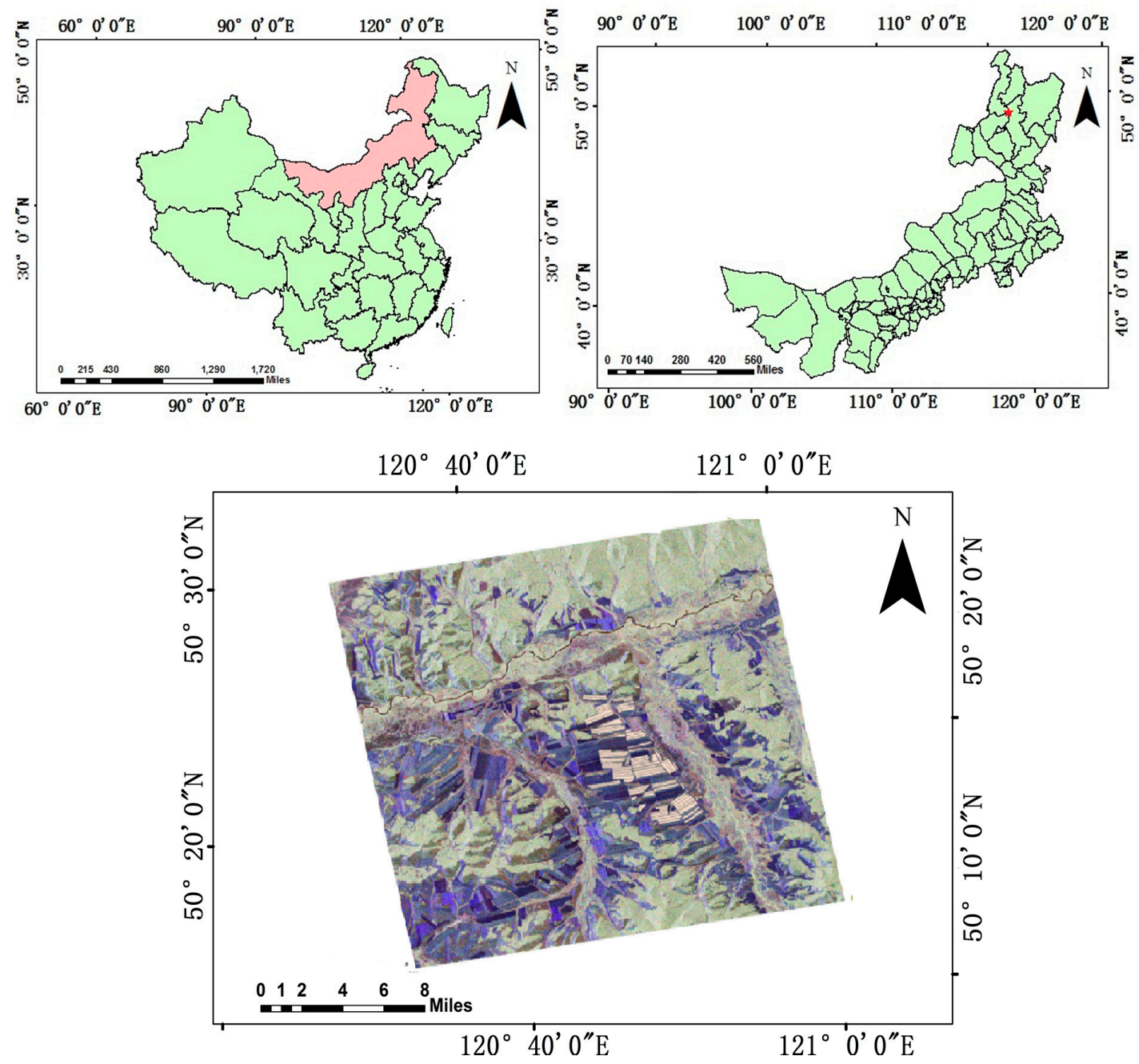

Figure 1. Location map of the test site in Inner Mongolia, China. The top-left sub-panel shows the coverage of the Inner Mongolia Autonomous Region of China (in pink color), the top-right sub-panel show shows the location of test site as one red star over the map of the Inner Mongolia Autonomous Region of China. The bottom sub-panel shows all the rape parcels in test site with light pink color based on a Pauli-basis RGB image of Radarsat-2, acquired on 23 May 2013.

Table 2. Principal stages of rape and relative BBCH scales. P1, Principal Stage 1.

\begin{tabular}{ccc}
\hline Acquisition Date & BBCH Stages & Principal Scales (DAS) \\
\hline 23 May 2013 & Germination (0) & P1 [-7, 15] \\
16 June 2013 & Leaf development (1) and formation of side shoots (2) & P2 [16, 39] \\
10 July 2013 & Stem elongation (3),inflorescence emergence (5) and flowering (6) & P3 [40, 63] \\
3 August 2013 & Development of fruit (7) & P4 [64, 87] \\
27 August 2013 & Ripening (8) and senescence (9) & P5 [89, 110] \\
\hline
\end{tabular}

In this research project, five synchronous ground measurement campaigns were carried out at each satellite overpass with a lag of no more than one day. Forty-four parcels among the 101 rape fields in the 
test site were provided with sowing dates by a local farmer or a farmland manager; sowing dates of the left were calculated by the method proposed in the paper [27]. All of the sowing periods of the test site lasted from 8 May-31 May. All of the sizes of the sample parcels vary from 3.3 hectares- 47.0 hectares, and the average size is 18.6 hectares. In each campaign, we recorded 11-14 parcels with leaf area index (LAI), plant height, surface soil moisture and above-ground biomass, including fresh and dry per square meter, as representatives. We randomly measured three sample sites within each field by the LI-COR LAI-2200 instrument and the Field Scout TDR 300 (time domain reflectometry) Soil Moisture Meter in High Clay mode after its calibration for LAI and 7.5-cm volumetric water content measurement, respectively. Each biomass sample was collected in a square of $0.5 \mathrm{~m} \times 2$ rows, and the related global position system (GPS) positions were also recorded for better matching the SAR data later. We also took digital photographs for visually recording rape development stages (Figure 2).

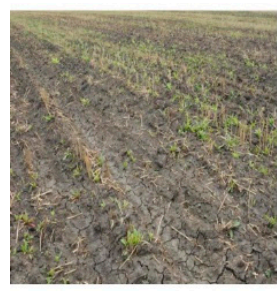

P1

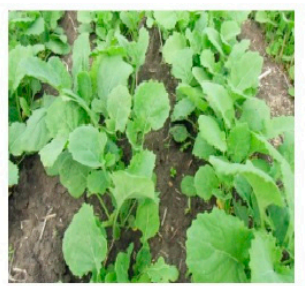

P2

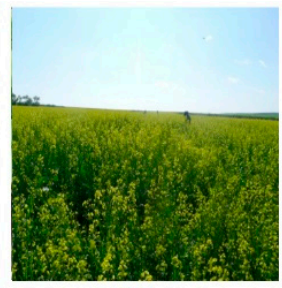

P3

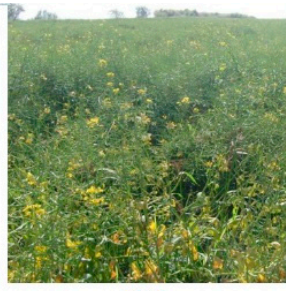

P4

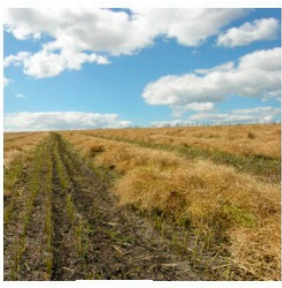

P5

Figure 2. Rape morphology at different stages in the test site.

\section{FP SAR Data Processing and Compact Polarimetric SAR Data Simulation}

\subsection{FP SAR Data Processing}

SAR data processing flowchart is shown as STAGE1 in Figure 3. Radarsat-2 FP SLC data were provided as separated real in-phase component (I) and the imaginary quadrature component (Q) for each polarization $(\mathrm{HH}, \mathrm{HV}, \mathrm{VH}$ and VV) in tiff image file format of 32 bits signed integer. The tiff data files for the four polarizations were imported into PolSARPro software (Version 5.0, from the European Space Agency software) as Sinclair matrix S2 shown by Equation (1), and then, the S2 matrix was radiometrically calibrated and calculated into the $\mathrm{C} 3$ matrix. The details for $\mathrm{S} 2$ radiometric calibration and transformation into the C3 matrix are introduced as follows.

$$
\mathrm{S} 2=\left[\begin{array}{ll}
S_{H H} & S_{H V} \\
S_{V H} & S_{V H}
\end{array}\right]
$$

The sigma zero radiometric calibration function for distributed object is shown as Equation (2).

$$
\sigma^{0}=\left\langle\frac{\mathrm{I}^{2}+\mathrm{Q}^{2}}{\mathrm{~A}^{2}}\right\rangle
$$

Here, a represents the gains, which only changes in the range direction, provided as a look up table in XML file format. Equation (1) is intended for calibrating the backscattering intensity; we should keep the phase of the input SLC data unchanged during the calibration processing in order to do further PolSAR data analysis. Therefore, we use A to scale both the I and Q signal of each polarization as shown in Equation (3).

$$
\mathrm{S} 2=\left[\begin{array}{ll}
S_{H H} & S_{H V} \\
S_{V H} & S_{V H}
\end{array}\right]=\left[\begin{array}{cc}
\frac{I_{H H}}{A}+i \frac{Q_{H H}}{A} & 0.5\left[\left(\frac{I_{H V}}{A}+i \frac{Q_{H V}}{A}\right)+\left(\frac{I_{V H}}{A}+i \frac{Q_{V H}}{A}\right)\right] \\
0.5\left[\left(\frac{I_{H V}}{A}+i \frac{Q_{H V}}{A}\right)+\left(\frac{I_{V H}}{A}+i \frac{Q_{V H}}{A}\right)\right] & \frac{I_{V V}}{A}+i \frac{Q_{V V}}{A}
\end{array}\right]
$$

Because Radarsat-2 data are acquired in mono-static mode, so in Equation (3), we average $S_{H V}$ and $S_{V H}$ to keep them equal. The symmetric S2 matrix was converted to a Hermitian positive semi-definite 
covariance matrix (C3) by multi-looking of the S2 matrix with two looks in both the azimuth and range direction using Equation (4).

$$
\mathrm{C} 3=\left[\begin{array}{lll}
\frac{1}{4} \sum_{\mathrm{i}=1}^{2} \sum_{\mathrm{j}=1}^{2} S_{H H \mathrm{i}, j}{ }^{2} & \sqrt{2} \frac{1}{4} \sum_{\mathrm{i}=1}^{2} \sum_{\mathrm{j}=1}^{2} S_{H H_{\mathrm{i}, j}} S_{H V_{\mathrm{i}, j}}^{*} & \frac{1}{4} \sum_{\mathrm{i}=1}^{2} \sum_{\mathrm{j}=1}^{2} S_{H H_{\mathrm{i}, j}} S_{V V_{\mathrm{i}, j}}^{*} \\
\sqrt{2} \frac{1}{4} \sum_{\mathrm{i}=1}^{2} \sum_{\mathrm{j}=1}^{2} S_{H H_{\mathrm{i}, j}}^{*} S_{H V_{\mathrm{i}, j}} & 2 \frac{1}{4} \sum_{\mathrm{i}=1}^{2} \sum_{\mathrm{j}=1}^{2} S_{H V_{\mathrm{i}, j}}^{2} & \sqrt{2} \frac{1}{4} \sum_{\mathrm{i}=1}^{2} \sum_{\mathrm{j}=1}^{2} S_{H V_{\mathrm{i}, j}} S_{V V_{\mathrm{i}, j}}^{*} \\
\frac{1}{4} \sum_{\mathrm{i}=1}^{2} \sum_{\mathrm{j}=1}^{2} S_{H H_{\mathrm{i}, j}}^{*} S_{V V_{\mathrm{i}, j}} & \sqrt{2} \frac{1}{4} \sum_{\mathrm{i}=1}^{2} \sum_{\mathrm{j}=1}^{2} S_{H V_{\mathrm{i}, j}}^{*} S_{V V_{\mathrm{i}, j}} & \frac{1}{4} \sum_{\mathrm{i}=1}^{2} \sum_{\mathrm{j}=1}^{2} S_{V V_{\mathrm{i}, j}}^{2}
\end{array}\right]
$$

The C3 matrix was then filtered by a $5 \times 5$ boxcar filter to further suppress speckle. The filtered C3 matrix was ortho-rectified using Mapready software with 30-m ASTER GDEM into the Universal Transverse Mercator (UTM) map projection. For more details about this SAR data processing procedure, the reader is referred to our previous work [27].

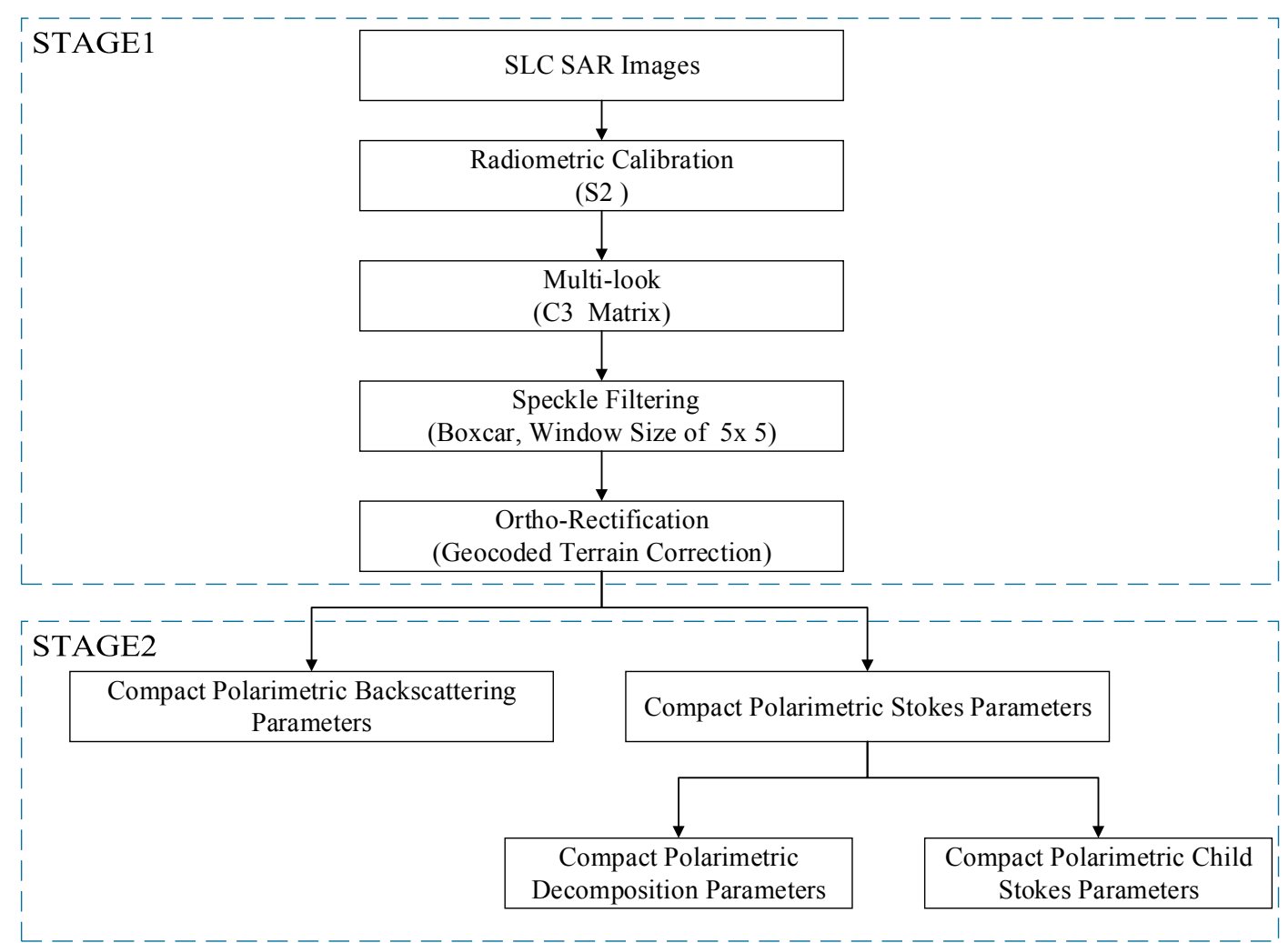

Figure 3. Fully-polarimetric (FP) SAR image processing, Compact polarimetric SAR images' simulation and related parameters' extraction.

\subsection{Compact Polarimetric SAR Data Simulation}

FP systems, which alternately transmit two orthogonal polarizations and record both received polarizations, suffer from the increases of the pulse repetition frequency and data ratio that cause the limitation of the associated energy budget, data storage and downlink requirement. To avoid these constraints of FP systems, CP, transmitting only a single polarization and receiving two orthogonal polarizations, has been proposed [17]. There are three CP modes named $\pi / 4$ mode, dual circular polarimetric (DCP) mode and circular transmits while linear receive (CTLR) mode, respectively [21]. Some investigations have demonstrated that the two circular transmit (DCP and CTLR) modes significantly outperform the $\pi / 4$ mode. The physical reason for the result is that the single linearly-polarized transmit of the $\pi / 4$ mode is not able to excite a target response for linear 
structures that are oriented orthogonal to the incident electric field, while DCP and CTLR work well in these areas. Even DCP and CTLR are not able to excite a target response in helical structures with their helicity oriented opposite that of the incident radiation; it does not affect their better performance on full interrogation of polarization states of typical terrain and manmade features because typical terrain tends to be composed more of linear structures (tree trunks and branches, buildings, fence posts, etc.) than helical structures (plowed fields viewed at near $45^{\circ}$ ). Some researchers believe that DCP mode is the linear combination of the CTLR mode, and both of them have the same decomposition characteristics [17]. For the above-mentioned reasons, in this paper, most of CP parameters were extracted from CTLR mode, and only the backscatter of RR (Right circular transmit and right circular receive) and RL (Right circular transmit and left circular receive)were extracted from DCP mode; the $\pi / 4$ mode has not been analyzed further in the paper. CP SAR data were simulated from FP Radarsat-2 data using elements in their C3 matrixes. The method for CP SAR data simulation was developed by Raney in 2007 [18], and its main procedures are introduced as follows.

At first, right circular transmission and linear orthogonal receivers were assumed for the CTLR $\mathrm{CP}$ mode in this paper. Based on the scene of FP described by the $2 \times 2$ Sinclair matrix [S], the electric vector $E_{R}$ of the backscattered field generated by right-circular illumination $R$ was evaluated as Equation (5):

$$
\begin{gathered}
E_{R}=[S] R \\
\text { where }[S]=\left[\begin{array}{ll}
S_{H H} & S_{H V} \\
S_{V H} & S_{V V}
\end{array}\right], R=\frac{1}{\sqrt{2}}\left[\begin{array}{c}
1 \\
-j
\end{array}\right]
\end{gathered}
$$

Then, the related $E_{H}$ and $E_{V}$ vectors of the single-look complex image were derived through the product of the linearly-horizontal and vertical polarized receiver, and they are described here by Equation (6).

$$
\begin{aligned}
& E_{H}=\left[\begin{array}{ll}
1 & 0
\end{array}\right] E_{R}=1 / \sqrt{2}\left(S_{H H}-j S_{H V}\right) \\
& E_{V}=\left[\begin{array}{ll}
0 & 1
\end{array}\right] E_{R}=1 / \sqrt{2}\left(S_{H V}-j S_{V V}\right)
\end{aligned}
$$

Four Jones matrix elements $\left(J_{11}, J_{12}, J_{21}, J_{22}\right)$ were calculated from $\left[\begin{array}{cc}E_{H} & E_{V}\end{array}\right]$ as Equation Group (7).

$$
\begin{gathered}
J_{11}=1 / 2\left(\left\langle\left|S_{H H}\right|^{2}\right\rangle+\left\langle\left|S_{H V}\right|^{2}\right\rangle+j\left\langle S_{H H} S_{H V}^{*}\right\rangle-j\left\langle S_{H V} S_{H H}^{*}\right\rangle\right) \\
J_{12}=1 / 2\left(\left\langle S_{H H} S_{H V}^{*}\right\rangle-\left\langle S_{H V} S_{H H}^{*}\right\rangle-j\left\langle\left|S_{H V}\right|\right\rangle^{2}-j\left\langle S_{H H} S_{V V}^{*}\right\rangle\right) \\
J_{21}=J_{12}^{*} \\
J_{22}=1 / 2\left(\left\langle\left|S_{V V}\right|^{2}\right\rangle+\left\langle\left|S_{H V}\right|^{2}\right\rangle+j\left\langle S_{V V} S_{H V}^{*}\right\rangle-j\left\langle S_{H V} S_{V V}^{*}\right\rangle\right)
\end{gathered}
$$

Then, the four CP Stokes parameters $\left(g_{0}, g_{1}, g_{2}, g_{3}\right)$ were derived from Jones parameters:

$$
\begin{gathered}
g_{0}=J_{11}+J_{22} \\
g_{1}=J_{11}-J_{22} \\
g_{2}=\operatorname{Re}\left\{\left\langle S_{H H} S_{H V}^{*}\right\rangle+\left\langle S_{H V} S_{V V}^{*}\right\rangle\right\}-\operatorname{Im}\left\langle S_{H H} S_{V V}^{*}\right\rangle \\
g_{3}=-\operatorname{Im}\left\{\left\langle S_{H H} S_{H V}^{*}\right\rangle-\left\langle S_{H V} S_{V V}^{*}\right\rangle\right\}-\operatorname{Re}\left\langle S_{H H} S_{V V}\right\rangle+\left\langle\left|S_{H V}\right|^{2}\right\rangle
\end{gathered}
$$

C3 elements were used to substitute the elements in Equation (8) and acquired the simulated CP data from original FP data.

$$
\begin{gathered}
g_{0}=1 / 2 C_{11}+1 / 2 C_{32}+1 / 2 C_{33}+(1 / \sqrt{2}) \operatorname{Im} C_{12}-(1 / \sqrt{2}) \operatorname{Im} C_{23} \\
g_{1}=1 / 2 C_{11}-1 / 2 C_{33}+1 / 2 C_{33}+(1 / \sqrt{2}) \operatorname{Im} C_{12}-(1 / \sqrt{2}) \operatorname{Im} C_{23} \\
g_{2}=(1 / \sqrt{2}) \operatorname{Re} C_{12}+(1 / \sqrt{2}) \operatorname{Re} C_{23}+\operatorname{Im} C_{13} \\
g_{3}=(1 / \sqrt{2}) \operatorname{Im} C_{12}-(1 / \sqrt{2}) \operatorname{Im} C_{23}+\operatorname{Re} C_{13}-\operatorname{Im} C_{22}
\end{gathered}
$$


For mathematical details of these procedures, the reader is referred to Raney (2007) [17].

Note that, although CP was derived from FP data, it only includes part of the information of FP. From Equation (5), we can see that only right circular Jones vectors were used for getting the transmit signal by the product between the FP scattering matrix and circular Jones vector. Therefore, the covariance of FP has four dimensions, but for $\mathrm{CP}$, it has two dimensions.

\section{Approach and Methods}

\subsection{Extraction of Compact Polarimetric Parameters}

Due to the objective of interpreting $\mathrm{CP}$ data reasonably and effectively, different interpretation methods were proposed by extracting different $\mathrm{CP}$ observations with their different natures, like the Stokes methods proposed by Raney $[17,18,28]$, the entropy and scattering angle alpha decomposition methods proposed by Cloude [24], and so on. According to the extraction methods or the related physical scattering mechanisms of these extracted $\mathrm{CP}$ parameters, in these papers, all of the $\mathrm{CP}$ observations were divided into four groups, named Stokes parameters, Stokes child parameters, backscattering parameters and decomposition parameters.

\subsubsection{Stokes Parameters}

Stokes parameters, which were proposed by Stokes in 1851 [29] and could be represented by four real numbers $\left(g_{0}, g_{1}, g_{2}, g_{3}\right)$, were proven to be a very convenient means to describe the received polarization state, as well as the scattering properties of targets. Taking circular transmission, dual polarization base at the receiver as an example, the four Stokes parameters are shown in Equation (10); for the detailed interpretation of the parameters in Equation (10), the reader is referred to [28]:

$$
\begin{gathered}
g_{0}=\left\langle\left|E_{R H}\right|^{2}+\left|E_{R V}\right|^{2}\right\rangle=\left\langle\left|E_{R L}\right|^{2}+\left|E_{R R}\right|^{2}\right\rangle \\
g_{1}=\left\langle\left|E_{R H}\right|^{2}-\left|E_{R V}\right|^{2}\right\rangle=2 \operatorname{Re}\left\langle E_{R L} E_{R R}{ }^{*}\right\rangle \\
g_{2}=2 \operatorname{Re}\left\langle E_{R H} E_{R V}{ }^{*}\right\rangle=2 \operatorname{Im}\left\langle E_{R L} E_{R R}{ }^{*}\right\rangle \\
g_{3}=2 \operatorname{Im}\left\langle E_{R H} E_{R V}{ }^{*}\right\rangle=\left\langle\left|E_{R L}\right|^{2}-\left|E_{R R}\right|^{2}\right\rangle
\end{gathered}
$$

This paper focuses more on the left side of the CTRL mode, and the Stokes parameters and their child parameters are also calculated from the left side. The Stokes parameters, which describe the state of a wave, can be calculated from equations introduced in Section 3.2; here, we focus more on their physical interpretations and relationship with four real polarization channels of FP, which measures the scattering of a wave. The complex scattering matrix S2 of CTRL mode in Equation (11) interpreted the relationship between four FP polarization channels and CP data. In most analyses of CP, Stokes parameters were more useful; because when we consider multilooking or speckle filtering, the average of Stokes parameters were entirely equivalent to forming the $2 \times 2$ average wave coherence matrix $\mathrm{C} 2$, as defined in (12). According to (11) and (12), we can build the relationship between each Stokes parameter and four FP polarization channels. That is why we can use Stokes parameters to describe the scattering characteristics of targets. Moreover, $g_{0}$ is the square of the amplitude of the reflected electric field vector, which is equal to the total power. $g_{1}$ reflects the tendency of the polarization to be more vertical $\left(g_{1}>0\right)$ or horizontal $\left(g_{1}<0\right)$. $g_{2}$ expresses the tendency to be polarized at $45^{\circ}\left(g_{2}>0\right)$ or $135^{\circ}\left(g_{2}<0\right) . g_{3}$ describes the left-handed polarization $\left(g_{3}>0\right)$ or right-handed polarization $\left(g_{3}<0\right)$. In this paper, the evolution of the four Stokes parameters was represented as a function of the DAS, which will be introduced later in the figures of Section 5. This type of figure will be used in this paper to analyze the dependence of all $\mathrm{CP}$ observables upon DAS.

$$
\left[\begin{array}{c}
E_{H C} \\
E_{V C}
\end{array}\right]=\frac{1}{\sqrt{2}}\left[\begin{array}{ll}
S_{H H} & S_{H V} \\
S_{V H} & S_{V V}
\end{array}\right]\left[\begin{array}{c}
1 \\
-i
\end{array}\right]=\frac{1}{\sqrt{2}}\left[\begin{array}{c}
S_{H H}-i S_{H V} \\
S_{V H}-i S_{V V}
\end{array}\right]
$$




$$
\mathrm{C} 2=\left[\begin{array}{cc}
\left\langle E_{H C} E_{H C}^{*}\right\rangle & \left\langle E_{H C} E_{V C}^{*}\right\rangle \\
\left\langle E_{V C} E_{H C}^{*}\right\rangle & \left\langle E_{V C} E_{V C}^{*}\right\rangle
\end{array}\right]=\frac{1}{2}\left[\begin{array}{cc}
g_{0+} g_{1} & g_{2+} i g_{3} \\
g_{2-} i g_{3} & g_{0-} g_{1}
\end{array}\right]
$$

\subsubsection{Stokes Child Parameters}

Raney also developed several useful child Stokes parameters from Stokes parameters with coherent dual-polarized SAR data, which can also be extracted from CP SAR data [11]. These parameters include the degree of polarization $(\mathrm{m})$, the degree of depolarization $(1-\mathrm{m})$; both $\mathrm{m}$ and $1-\mathrm{m}$ describe the state of polarization; if $\mathrm{m}$ is zero, we have depolarized wave; at the other extreme, when $\mathrm{m}$ is one, then we have a purely polarized wave. $1-\mathrm{m}$ is inversed. The phase difference $(\delta)$ is the phase between the linear components of the wave. When $\delta>0$, surface scattering is dominant; when $\delta<0$, odd scattering is greater than surface scattering. The circular degree angle $(\chi)$, which is the supplementary angle of scattering angle $\alpha$, has the inverse meaning of $\alpha$; readers are referred to the details in $[13,24]$. The degree of linear polarization $\left(P_{l}\right)$ and the degree of circular polarization $\left(P_{c}\right)$ are two parameters describing how much wave power was linearly polarized or circularly polarized by the target scattering. The circular polarization ratio $\left(U_{c}\right)$ is the traditional indicator of frozen volatile deposits. It is an explicit ratio of the image power in the opposite-sense circular polarization, under the condition that transmitted polarization is circular. Additionally, the linear polarization ratio $\left(U_{l}\right)$ is similar to $U_{c}$, but it describes the ratio of linear polarization. For the equations or calculation details of these parameters, readers are referred to $[17,18]$.

\subsubsection{Backscattering Parameters}

Previous studies had also demonstrated that the CP backscattering parameters play an important role in crop phenology stages' inversion [3], crop mapping [21] and crop growth parameters' inversion [25]. This paper simulated 4 backscattering parameters $\left(\sigma_{\mathrm{RH}}, \sigma_{\mathrm{RV}}, \sigma_{\mathrm{RL}}, \sigma_{\mathrm{RR}}\right)$ from the fully-polarized scattering matrix $([S])$ and a ratio parameter $\left(\sigma_{\mathrm{RV}} / \sigma_{\mathrm{RH}}\right)$ to inverse the rape growth parameters. The calculation details for these four backscattering parameters are from Equation (2).

\subsubsection{Decomposition Parameters}

Polarimetric decomposition is a popular and effective way for radar remote sensing to extract physical information from the observed scattering for the terrain types $[7,10]$. There are three $\mathrm{CP}$ decomposition algorithms named $\mathrm{m}-\delta$ decomposition, $\mathrm{m}-\alpha$ decomposition and $\mathrm{m}-\chi$ decomposition [10]. However, $\alpha$ is a supplementary angle for $\chi$. Therefore, in this paper, only $\mathrm{m}-\delta$ and $\mathrm{m}-\alpha$ decomposition were introduced. $\mathrm{m}-\delta$ decomposition, which was proposed by Raney [17], is based on Stokes parameters. In this expression, $m$ is the polarization degree, and $\delta$ is the phase between the linear components of the wave. $\mathrm{m}$ is a special characteristic of the partial polarimetric wave; here, it is used for the distinction of polarized and unpolarized signals. Since $\delta$ is sensitive to the surface scattering and double-bounce scattering, it is used for the distinction of these two scattering mechanisms. $\mathrm{m}-\delta$ decomposition was generated as three components $\left(P_{D \delta}, P_{S \delta}, P_{V \delta}\right) . P_{V \delta}$ is related to dominantly-depolarized backscatter (e.g., low degree of polarization) which characterized vegetation medium in practice. $\delta$ helps with distinguishing the dominant scattering mechanism between double-bounce $\left(\delta<0\right.$ thus $\left.P_{S \delta}<P_{D \delta}\right)$ and surface scattering $\left(\delta>0\right.$ thus $\left.P_{S \delta}>P_{D \delta}\right)$. $\mathrm{m}-\alpha$ decomposition, proposed by Cloude in 2012 [24,30], is based on entropy/alpha and RVoG (random volume over ground) or OVoG (oriented volume over ground) models. The three components of $\mathrm{m}-\alpha$ decomposition are $P_{D \alpha}, P_{S \alpha}$ and $P_{V \alpha}$. Similar to $\mathrm{m}-\delta$ decomposition, $P_{V \alpha}$ is related to dominantly-depolarized backscatter (e.g., low degree of polarization), which characterized vegetation medium in practice. $\alpha$ helps with distinguishing the dominant scattering mechanism between double-bounce $\left(\pi / 4>\alpha>0\right.$ thus $\left.P_{S \alpha}>P_{D \alpha}\right)$ and surface scattering $\left(\pi / 2>\alpha>\pi / 4\right.$ thus $\left.P_{S \alpha}<P_{D \alpha}\right)$. In these two CP decomposition methods, the volume scattering components are the same, so only one parameter was picked from $P_{V \delta}$ and $P_{V \alpha}$ and analyzed 
in this paper. Some previous studies also show several ratios like volume scattering/total scattering $\left(r_{1}\right)$, double-bounce scattering/total scattering $\left(r_{2}\right)$, surfacing scattering/total scattering $\left(r_{3}\right)$ and volume scattering/(double-bounce scattering + surface scattering) $\left(r_{4}\right)$, which were useful in distinguishing crop phenology and crop productivity mapping [21]. In this paper, we also extracted these ratios from CP data.

\subsection{Random Forest Regression Algorithm for Rape Growth Parameters Inversion}

Random forests (RF) were introduced by Leo Breiman [31] in 2001; they can be used for either a categorical response variable, like "classification", or a continuous response, referred to as "regression". The appealing characteristics of RF for parameter prediction include its relatively fast speed for training and prediction, its capability for direct use for high-dimensional problems or parameters, its measurement of variable importance simultaneously and its dependence on small parameters [26]. The random forest is a tree-based ensemble with each tree depending on a collection of random variables. A tree represents a set of conditions or restrictions that are hierarchically organized and successively applied from a root to a leaf of the tree. RF begins with several bootstrap samples, which are in accord with the number of the original training dataset and are randomly drawn from the original training dataset. Then, a set of regression trees is created to fit each bootstrap sample from the original data respectively. For each node per tree, a small set of input variables selected from the total set is randomly considered for binary partitions. The splitting criterion is based on "goodness of fit" defined in [32] for regression. The procedure continues recursively until a stopping criterion is met. In this paper, for example, the procedure stops when all un-split nodes contain fewer than the fixed number of cases. When the stopping criterion is met, un-split nodes are called "terminal nodes", which form the final partition of the predictor space of the regression trees. The predicted value is calculated by averaging over all of the regression trees. If the samples are fixed, there are two parameters that play the substantial roles in the RF regression algorithm. They are the number of regression trees and the number of input variables per node [32].

In this paper, we used the Random Forest package in the R software for our studies. The number of regression trees (ntree in R) was optimized using the training dataset and RMSE to find the values that could best predict the growth parameters. The number of input variables per node (mtry in R) was tested from 1/3 to the whole number of CP parameters, and then, the mtry values yielding the lowest RMSE were selected. Meanwhile, in this study, SAR observations at P1 contained little vegetation information, and at P5, as the crop entered into the productive and harvest stage, which seemed to be too late for farm practice, also showed fluctuated values for most of the CP parameters; as a result, only P2, P3 and P4 were stable and selected for the inversion of growth parameters [27].

\section{Results}

\subsection{Analysis of CP Observables' Sensitivity to Rape Growth Parameters}

Both the temporal evolution of CP response and rape growth parameters during the whole growth cycle were analyzed as a function of the number of day after sowing (DAS). Their change trends were also compared with the objective to find the sensitive CP parameters for growth parameters and to analyze the scattering mechanisms of rape at different growth stages. At each principal scale, DAS of rape fields were calculated with the methods proposed in [27]; CP observables were averaged from rape fields with the same DAS. Growth parameters like LAI, stem height and biomass and related DAS were surveyed in each campaign with 11-14 representative rape fields. Figure 4 shows the plots of the growth parameters as a function of DAS. LAI, stem height and biomass of rape were collected from 16 June, when the plants emerged. LAI kept increasing before DAS of 45, and the points formed the first peak at DAS of 45. Then, it showed an obviously fast decrease from DAS of 45-65. The curve of the scatter plots began to increase again from DAS of 65 and formed the second peak at DAS of 80 . Then, the curve ended in a fast decreasing slope that obtained the value of 2.3 at DAS of 88 . The highest 
LAI value, which was located at the second peak, is 3.5. The decrease of LAI between DAS of 45 and 65 resulted from the falling and withering of large leaves in the bottom canopy layer of rape beginning at that time, which resulted in smaller and less leaves in a unit volume. Stem height kept increasing before DAS of 75, then the increasing tendency of the scatter plots tapered to a flat line until DAS $=95$. Biomass showed a gentle increase from DAS of 21-65, then there was a distinct bounce between DAS of 65 and 80. The points form a flat peak between DAS of 65 and 85; this meant saturation arose in biomass during this period. The curve of the scatter plot ended in a sharp decreasing slope that tapered to a flat platform after DAS of 85 .

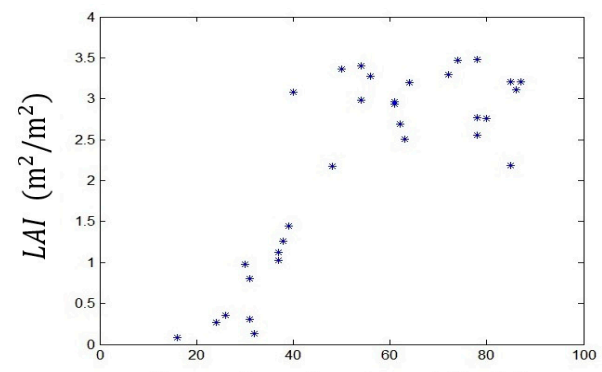

Day after Sowing (d) (a)

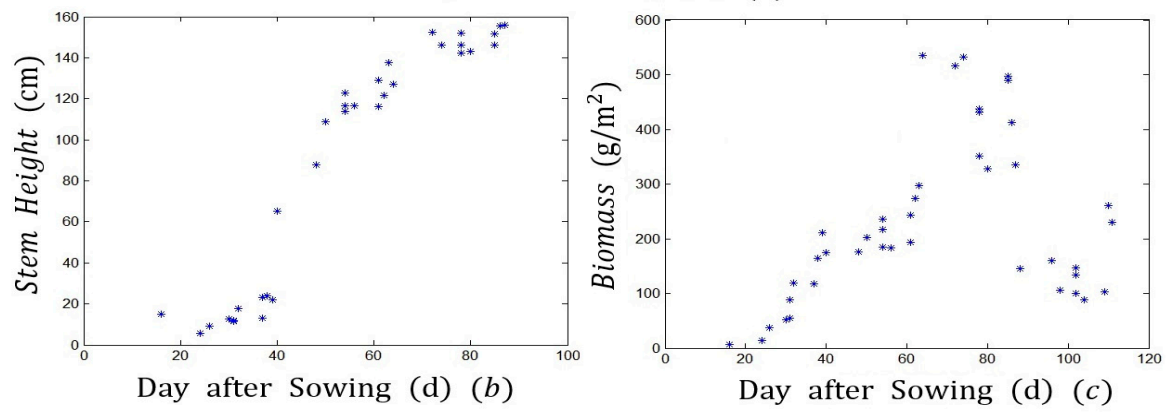

Figure 4. Temporal evolution of three growth parameters based on 101 fields over the whole growth cycle. (a) LAI; (b) stem height; (c) biomass. Each point in these plots is the average value of all fields having the same sowing date. The data were only collected after the emergence of the plants.

\subsubsection{Stokes Parameters}

In the first place, we observe in Figure $5 \mathrm{a}$ that $g_{0}$ presented five different principal ranges during the cycle, the trend of which was perfectly coincident with the FP span in our previous study in [27]. Figure $5 \mathrm{a}$ showed the lowest average point value of 0.05 during P1. During this stage, the fields were not sown or just sown; the rape leaves were just emerging through the soil surface with plant heights not exceeding $5 \mathrm{~cm}$ during this stage. The plants were so small that they were not visible to radar, and hence, the soil scattering dominated the SAR response. The SAR backscattering power was mainly influenced by soil roughness and moisture, which kept the backscattering power stable before the plants' emergence. This also explained the relatively same values of $g_{0}$ at P1. At P2, the scatter point values increased gently since the rape leaves developed and became larger $(6.6 \mathrm{~cm}$ average leaf width). These leaf sizes were comparable to C-band wavelength $(5.6 \mathrm{~cm})$, and thus, greater plant scattering resulted in the increasing values of $g_{0}$. As the plants developed to P3, the scatter points showed a gently decreasing trend that may result from the withering and falling of large leaves in the rape bottom canopy layer, which induced less leaves in a unit volume. This phenomenon was in line with LAI measurement in the field campaign, which decreased from $3.6(\mathrm{DAS}=41)$ to 2.5 (DAS = 63). At P4, there was an obvious jump of scatter point values; the highest value at this stage was 0.4 . It indicated that the pods of rape caused significant random scattering; at the same time, stem elongation of rape also caused the increase of dihedral response. Moreover, according to the precipitation and mean air temperature record collected in this research, there had been rainfall events at the end of July and the 
beginning of August, which also led to the bounce of the high scattering values. The rain effect on the backscattering of plant canopies had also been observed in a previous study [9]. However, the trend plotted in Figure 5a agreed with Figure 4c; hence, the information contained in such an observable was sensitive to biomass change. $g_{1}$ kept at zero during P1, began to increase gently at P2 and reached the highest value at the end of P3. The physical interpretation of $g_{1}$ from different stages can be found in the previous analysis of the backscattering powers of $\mathrm{HH}$ and $\mathrm{VV}$ polarizations [9]. At the beginning of the season, with the small plants, the Bragg scattering from the rough surface predicted $\mathrm{HH}$ as equal to $\mathrm{VV}$, then the developed plants grew their leaves in the horizontal direction, which generated the extinction of $\mathrm{HH}$ higher than VV and showed positive values of $g_{1}$ at P2 and P3. At P4, the emergence of double-bounce at P3 began strongly at P4 and led to high extinction of VV, which was higher than $\mathrm{HH}$ at P2 and P3; the scatter points showed negative values. At P5, some fields were harvested and plowed, which caused the fluctuation between the value of zero. When comparing Figure $5 \mathrm{c}$ and $\mathrm{d}$, it was clear that at the first three stage, $45^{\circ}$ polarization component was zero, but left-hand circular polarization kept increasing from P1 to the end of P2; it then began to decrease and reached about zero at the end of $\mathrm{P} 3$. The phenomenon was coincident with the $45^{\circ}$ and left-hand circular polarization continuing to increase from P1 to the end of P2; it then began to decrease and reached about zero at the end of $\mathrm{P} 3$. The phenomenon was coincident with the $45^{\circ}$ and circular polarization scattering mechanism. For instance, $45^{\circ}$ polarization is not able to excite a target response for linear structures that are oriented orthogonal to the incident electric field, and circular polarization is not able to excite a target response in helical structures. At the first three stages, the scattering was dominated by $\mathrm{HH}$ and VV, which matches the evolution of $g_{1}$. At Stage 4, dihedral scattering was strong and generated higher $45^{\circ}$ polarization scattering and right-hand circular polarization; these results were confirmed in a previous study of $\mathrm{CP}$ mode [23]. At the end stage, the reason for the fluctuation of the values of $g_{2}$ and $g_{3}$ was somehow analogous to $g_{1}$.
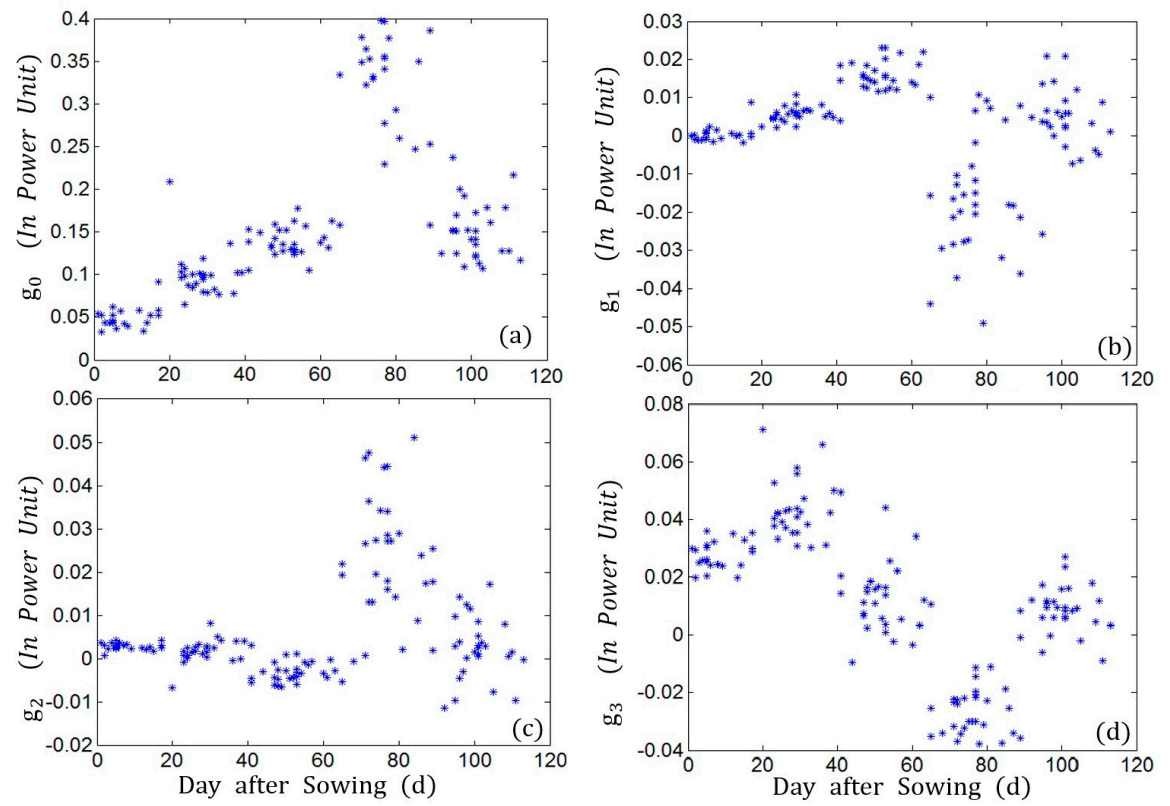

Figure 5. Temporal evolution of four Stokes parameters based on 101 fields over the whole growth cycle. (a) $g_{0} ;$ (b) $g_{1} ;$ (c) $g_{2} ;$ (d) $g_{3}$. Each point in these plots is the average value of all fields having the same sowing date. The data were collected during the whole growth cycle of rape.

\subsubsection{Stokes Child Parameters}

Some of the Stokes child parameters like $m, \delta, U_{c}$ and $P_{c}$ were known to be useful for crop mapping and monitoring $[9,21]$. In this paper, these observables were analyzed for the first time for growth 
parameters' inversion. Some of them present evident significance in terms of growth parameters as the function of DAS. Figure 6 present the eight Stokes child parameters. Since alpha (scattering angle) is useful for physical scattering mechanism interpretation, hence they were calculated from $\chi . \mathrm{m}$ and $1-\mathrm{m}$ had an inverse trend with respect to each other; this was also the case for $U_{c}$ and $P_{c}$; so here, only $\mathrm{m}$ and $U_{c}$ were analyzed as representative. $\mathrm{m}$ was related to entropy, i.e., the minimum $\mathrm{m}$ corresponds to maximum entropy. Therefore, the evolution of $\mathrm{m}$ can be jointly exploited with alpha. From the first two stages (P1 and P2), m kept decreasing from 0.7 down to 0.4, while the dominant alpha stays at $10^{\circ}$, denoting that surface scattering was the main contribution mechanism. Then, at P3, m lowered to 0.25 ; the dominant alpha was above $30^{\circ}$ and increasing to $50^{\circ}$, which corresponded to dihedral scattering according to [3,9]. This meant that at P3, the backscatter was dominated by double-bounce interaction between the stem and the surface. From P4 to the end of the growth cycle, when plants' scattering become more random than before, $\mathrm{m}$ kept at 0.23 , while alpha stayed at $50^{\circ}$ at $\mathrm{P} 4$ and decreased to $45^{\circ}$ at $\mathrm{P} 5$. This meant that it was difficult to distinguish the different stages with these two parameters. $m$ was sensitive to the growth parameters before the first three stages of the whole growth cycle. A stable platform of $\delta$ at the first two stages was observed in Figure 6c, which was quite different from the results reported in [9] where the values were quite noisy. There was almost no double-bounce scattering in the first two stages with the value of $\delta$ equal to $90^{\circ}$. At these two stages, rapes were just sown or began to emerge, and surface scattering was the dominant scattering mechanism, which includes both circular polarization and linear polarization. At $\mathrm{P} 3$, it became negative and noisy, which meant the double-bounce scattering resulted in attenuation of vertically-polarized return. This phenomenon was confirmed by the evolution of $P_{D \delta}$ and $P_{D \alpha}$ in decomposition parameter groups. At $\mathrm{P} 5$, this value became positive and noisy since the random scattering made the relative phase difference meaningless, but it also showed that the surface scattering component was higher than the double-bounce scattering component. The trend of $U_{c}$ was perfectly coincident with the results reported in [3]; meanwhile, it resembled perfectly the evolution of three growth parameters during the whole rape growth cycle, especially with biomass. $P_{l}$ was quite noisy during the entire growth cycle; this meant the total linear polarized component kept stable during the growth cycle. Such behavior was due to the similar surface scattering power during the whole cycle. It was confirmed by the evolution of the surface scattering components like m-delta-s and m-alpha-s in decomposition parameter groups. Comparing Figures $5 \mathrm{~d}$ and $6 \mathrm{~g}$, the inverse of the $U_{l}$ trend resembled the $g_{3}$ well, but with a different scale. Therefore, $U_{l}$ was also sensitive to different growth stages, but insensitive to the growth parameters.
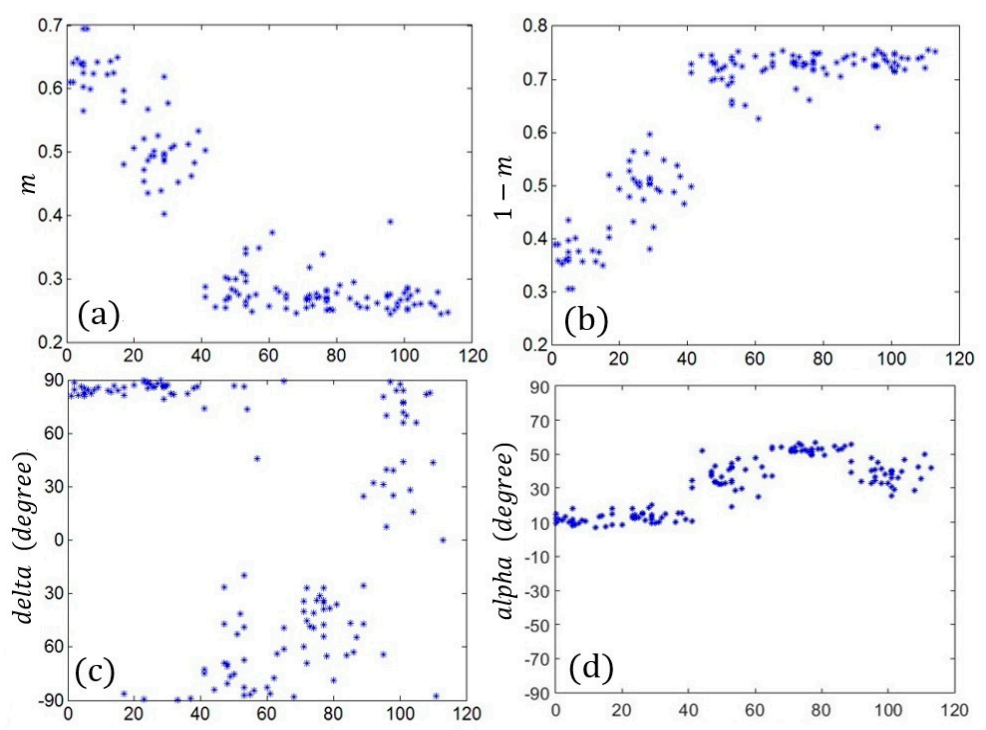

Figure 6. Cont. 

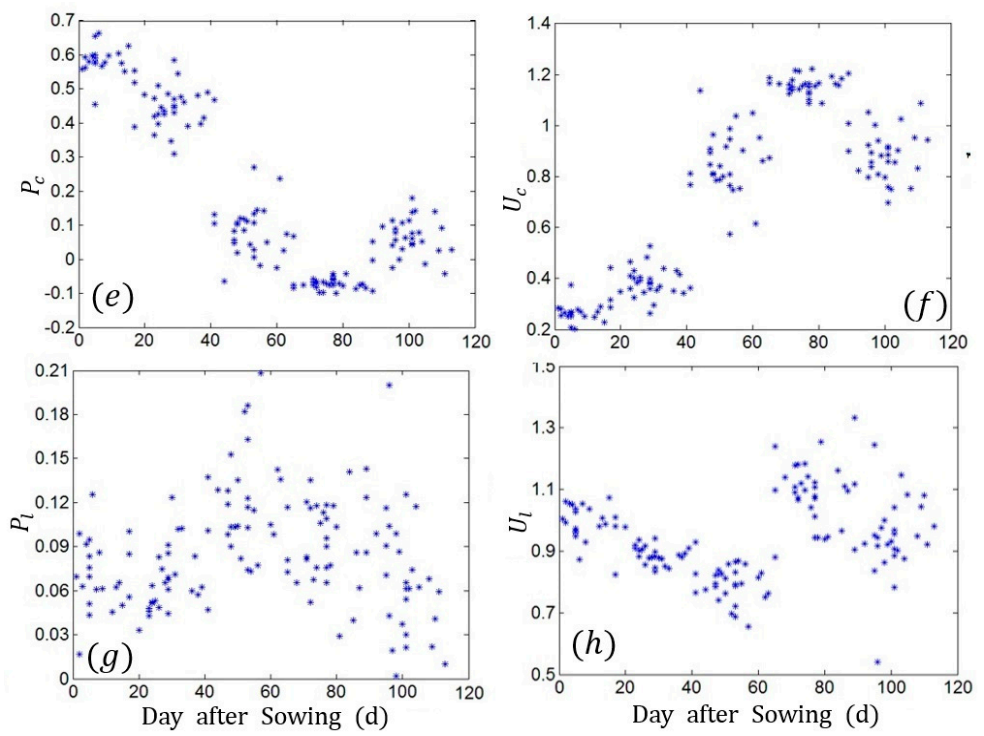

Figure 6. Temporal evolution of four Stokes parameters based on 101 fields over the whole growth cycle. (a) $\mathrm{m} ;(\mathbf{b}) 1-\mathrm{m} ;$ (c) $\delta$; (d) $\alpha$; (e) $P_{c} ;(\mathbf{f}) U_{c} ;(\mathbf{g}) P_{l} ;$ (h) $U_{l}$. Each point in these plots is the average value of all fields having the same sowing date. The data were collected during the whole growth cycle of rape.

\subsubsection{Backscattering Parameters}

The evolution of backscattering coefficients is not new and constitutes the core of most studies of radar remote sensing for crops' mapping and monitoring [33,34]. They had been included here as a reference for comparison with other $\mathrm{CP}$ parameters and also the backscattering coefficients of FP data, so only four observables were shown here as representatives. All backscatter powers increased from very low values at the first stage with a gentle slope to the end of P2. There was an obvious decline at P3, which resulted from the withering and falling of large leaves at the bottom of rape. The values reached their maximum value at $\mathrm{P} 4$ because of the strong double-bounce scattering and random scattering at this stage. Then, the values decreased to a moderate value at $\mathrm{P} 5$ with the leaves falling and rape harvesting. Figure $7 \mathrm{c}$ describes the difference between the response at RH and RV by their ratio. RV started from values similar to $\mathrm{RH}$ at the first stage and then kept higher until the end of P3. Then, at P4, a distinct jump was shown on P4 for the reason of high attenuation of vertically-polarized return. At the end stage, $\mathrm{RH}$ and $\mathrm{RV}$ showed similar values again for the dominated surface scattering mechanism. The behavior of RL and RR was in agreement with RH and RV. All of them were excellent indicators for different stages of rape and also had great potential for three growth parameters' inversion. 

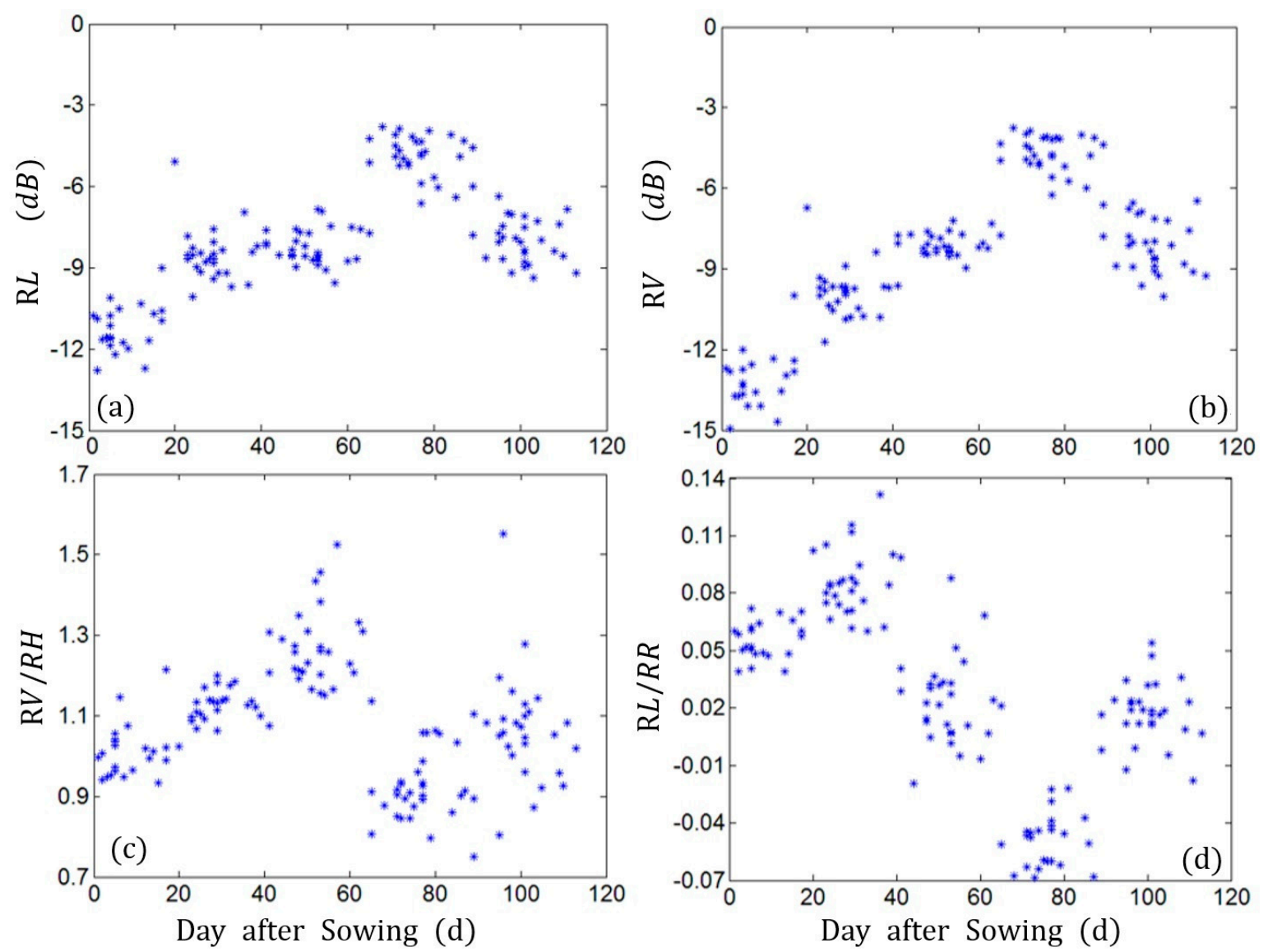

Figure 7. Temporal evolution of four two backscattering parameters and two ratio of backscattering coefficients based on 101 fields over the whole growth cycle. (a) RL(right cicular transmit and left circular recieve) (b) RV(right cicular transmit and verctical linear recieve); (c) RV/RH (the ratio between backscattering coefficient of channel RV and RH); (d) RL/RR (the ratio between backscattering coefficient of channel RL and RR). Each point in these plots is the average value of all fields having the same sowing date. The data were collected during the whole growth cycle of rape.

\subsubsection{Decomposition Parameters}

$\mathrm{m}-\delta$ and $\mathrm{m}-\chi$ decompositions had already been applied in rape biomass inversion in [25]; in this paper, we paid more attention to the difference between these two decomposition results and their sensitivity to the inversion of LAI, biomass and stem height. Since their volume scattering components are same, here, only one of them is shown in Figure 8c. According to previous research $[3,9,25]$, the ratios of different scattering components for full polarized data were also sensitive to crop growth cycle, so these observables for $\mathrm{CP}$ data are shown in Figure 8, Figure $8 \mathrm{~d}$ for the ratios of volume scattering with the summary of surface and dihedral scattering, Figure $8 \mathrm{~g}$ for the ratio of dihedral scattering with total scattering power, Figure $8 \mathrm{f}$ for the ratio of surface scattering with total scattering power. Since the ratio of volume scattering with total scattering power was the same as the evolution of $1-\mathrm{m}$ (Figure $7 \mathrm{~b}$ ), it is not shown here. Figure $8 \mathrm{a}, \mathrm{b}$ presents the evolution of $\mathrm{m}-\delta$ decomposition dihedral scattering and surface scattering respectively. (a) presents three different ranges during the cycle: lower and stable values during the first two stages (P1 and P2), reaching to the highest values during P3 and P4, decreasing and noisy at P5. (b) presents two different ranges during the cycle: noisy values during stage $\mathrm{P} 3$ and $\mathrm{P} 4$, others with stable values at y equal to $-8 \mathrm{~dB}$. On the contrary, the evolution of these two components of $\mathrm{m}-\alpha$ decomposition showed more sensitive trends during the entire growth cycle. The trend of surface scattering of $m-\alpha$ was perfectly coincident with the evolution of biomass, as well as the volume scattering component. Better performance of $m-\alpha$ 
was also confirmed in the literature [30]. The reason for the better performance of $m-\alpha$ was the insensitivity of $\alpha$ to the scattering direction angle of targets. Hence, the components for ratio calculation in Figure 8 were extracted from $\mathrm{m}-\alpha$ decomposition. This phenomenon was also confirmed by the $\delta$ values at the first two growth stages of rape, which almost equaled $90^{\circ}$. It could be the effects of direction angles within $\delta$. However, the comparison between volume scattering and dihedral, surface scattering showed the dominant volume scattering component during the entire cycle, which reveals the over-estimation of the volume scattering of this decomposition method. Figure $8 \mathrm{~d}$ confirms these over-estimations during the entire cycle, where the ratio was higher than one after P2. For these reasons, only $m-\alpha$ decomposition used for the interpretation of the crops' scattering mechanisms may cause ambiguity. Fortunately, with Stokes observables and their child parameters, this ambiguity has been solved, as demonstrated in the previous paragraphs. According to the mathematical details introduced in $[17,18,35]$, the evolution of the ratio of surface scattering with total power and the ratio of dihedral scattering with total power are similar to $P_{c}$ and $U_{c}$ of the Stokes child parameters, respectively. It has also been confirmed by the evolution trend shown in Figure $8 \mathrm{~g}$, $\mathrm{h}$ here. These two parameters also showed sensitivity to the growth parameters.
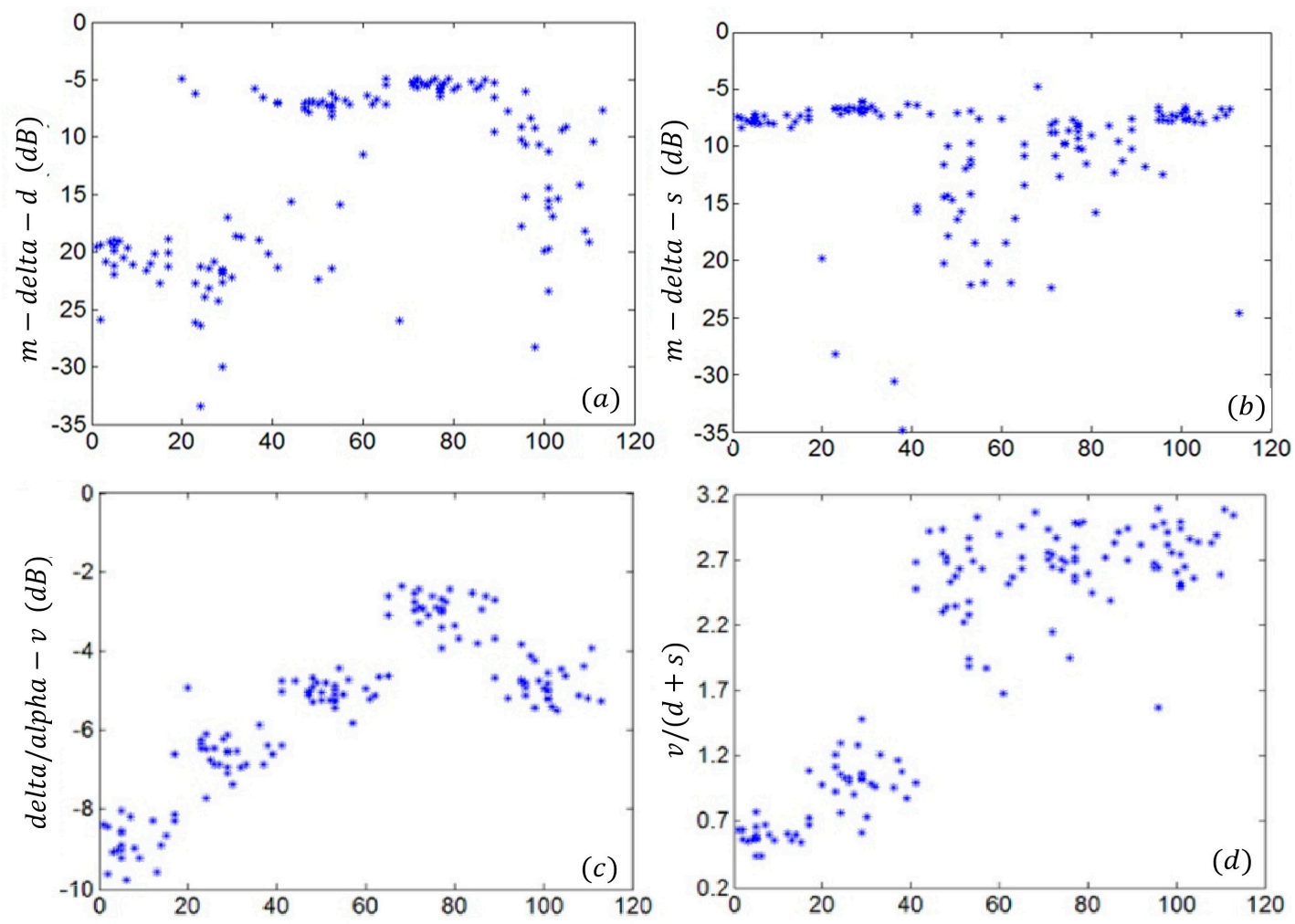

Figure 8. Cont. 

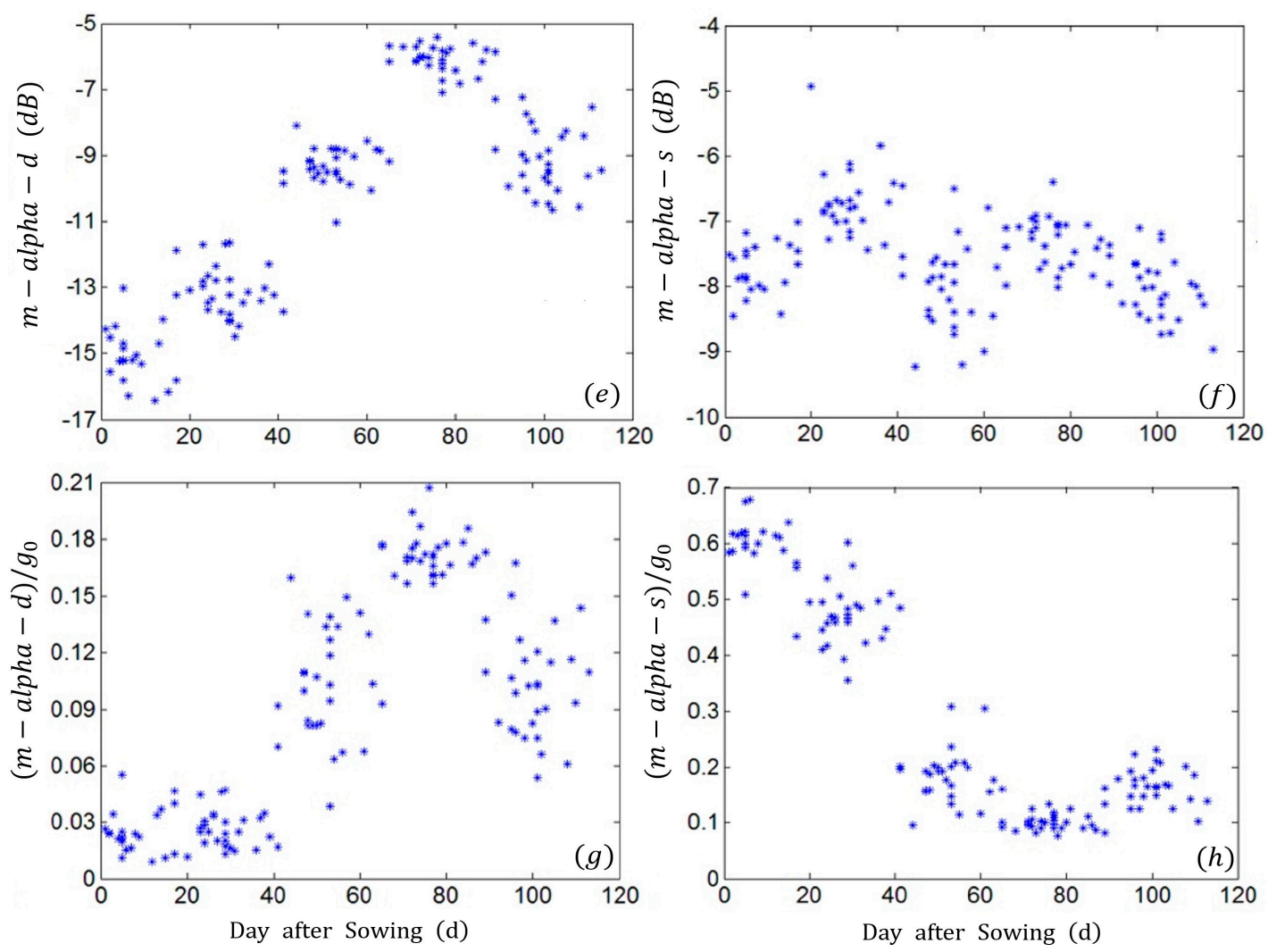

Figure 8. Temporal evolution of four decomposition parameters based on 101 fields over the whole growth cycle. (a) m-delta-d; (b) m-delta-s; (c) m-delta/alpha-v; (d) v/(d + s); (e) m-alpha-d; (f) m-alpha-s; (g) m-alpha-d $/ g_{0}$; (h) m-alpha-s $/ g_{0}$. Each point in these plots is the average value of all fields having the same sowing date. The data were collected during the whole growth cycle of rape.

\subsection{Growth Parameters Inversion with Random Forest}

In this paper, the main objective is to find the feasibility of the RF model for growth parameters with CP parameters, so the PF model was investigated in this paper for the inversion of these three growth parameters. The number of regression trees for RF here was decided according to the stable regression root mean square error (RMSE) with the given set of training input-output pairs (i.e., CP parameters: biomass/stem height/LAI). For these three growth parameters, ntree is 500 . At each node per tree, $m$ try is nine, which was randomly selected as $1 / 3$ from all $27 \mathrm{CP}$ parameters. The importance of each CP observable for the three growth parameters' inversion is also introduced through Figure 9. In Figure 9, we observe that some CP parameters like $g_{0}, g_{3}, P_{c} U_{c}$, etc., which showed the potential for the inversion of growth parameters physically according to the analysis in previous sections, also showed high importance in the RF algorithms. However, there were also other CP parameters like $g_{2}$ and $\delta$ for biomass inversion, $\mathrm{m}$ and $\alpha$ for stem height inversion, $g_{2}$ and surface scattering $\mathrm{m}-\alpha$-s for LAI inversion that showed inconspicuous coincidence with growth parameters, also showing high importance in RF. The phenomenon was interpreted as the valuable selection ability of RF in modeling for growth parameters inversion, especially in a nonlinear system, for which is commonly difficult to decide which variable to remove. It also confirmed that it was difficult for the physical interpretation of vegetation scattering mechanism for RF inversion models. Similar to the previous section, the 101 fields were divided into 44 groups according to their DAS for biomass inversion. The estimation model training was based on 33 average results, and 11 were left for validation. However, 101 fields were divided into 33 groups according to their DAS for stem height and LAI inversion because of the fewer field campaigns for stem height and LAI records. Twenty two groups in the total of 33 were chosen for model training, and the remaining 11 for validation. We use 
the determination coefficient $\left(R^{2}\right)$ and RMSE between the estimated results and field measured results to assess the inversion accuracy. Since regression models were popular for crop growth parameters inversion; we performed some similar regression models like the linear model, quadratic model, power model, index model and the logarithm model for growth parameters' inversion according to the sensitivity of $\mathrm{CP}$ parameters to the growth parameters, which were analyzed above. Among all of these models, the quadratic model had the best estimation accuracy and RMSE compared to the other models for all growth parameters. The best $\mathrm{CP}$ parameter for biomass inversion was $g_{3}$ and for stem height and LAI was $U_{c}$; the detail for $\mathrm{R}^{2}$ and RMSE is shown in Table 3. The optimal models and related equations for three growth parameters are shown in Figure 10. In Figure 10a is the best single parameter regression model for biomass; Figure 10b is the best single parameter regression model for stem height; Figure 10c is the best single parameter regression model for LAI. Table 3 describes the details of the estimation results. Table 3 shows the RF results at the main growth stages of P2, P3 and $\mathrm{P} 4$ for biomass, stem height and LAI. The validation test results described in Table 3 showed high estimation accuracy of RF for the three growth parameters. The determination coefficient $\left(R^{2}\right)$ between the estimation values and measured values for biomass was 0.93 with $R M S E=46.24 \mathrm{~g} / \mathrm{m}^{2}$. The $R^{2}$ for LAI is the highest among the three growth parameters, which was 0.96 with RMSE $=0.25$. The $\mathrm{R}^{2}$ for stem height is 0.95 with RMSE $=13.5 \mathrm{~cm}$. The relative significance level for all of the models was $p<0.001$.

Table 3. The details of the relationship between predicted and observed growth parameters.

\begin{tabular}{ccccc}
\hline $\begin{array}{c}\text { Growth } \\
\text { Parameters }\end{array}$ & RF-R2 & RF-RMSE & $\begin{array}{c}\mathbf{R}^{\mathbf{2}} \text { (Regression } \\
\text { Model) }\end{array}$ & $\begin{array}{c}\text { RMSE (Regression } \\
\text { Model) }\end{array}$ \\
\hline Biomass & 0.93 & $46.24 \mathrm{~g} / \mathrm{m}^{2}$ & 0.765 & $73.20 \mathrm{~g} / \mathrm{m}^{2}$ \\
Stem height & 0.95 & $13.5 \mathrm{~cm}$ & 0.923 & $18.71 \mathrm{~cm}$ \\
LAI & 0.96 & 0.25 & 0.857 & 0.66 \\
\hline
\end{tabular}

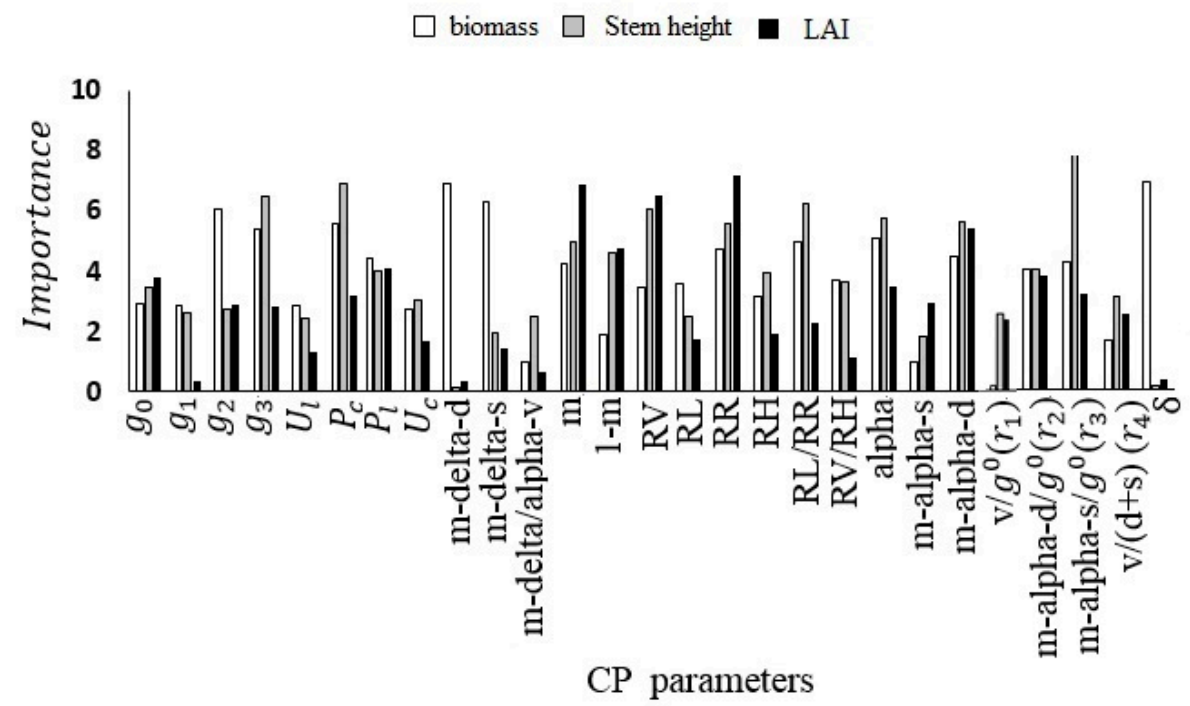

Figure 9. The order of importance for all of the $\mathrm{CP}$ parameters in the RF models. 


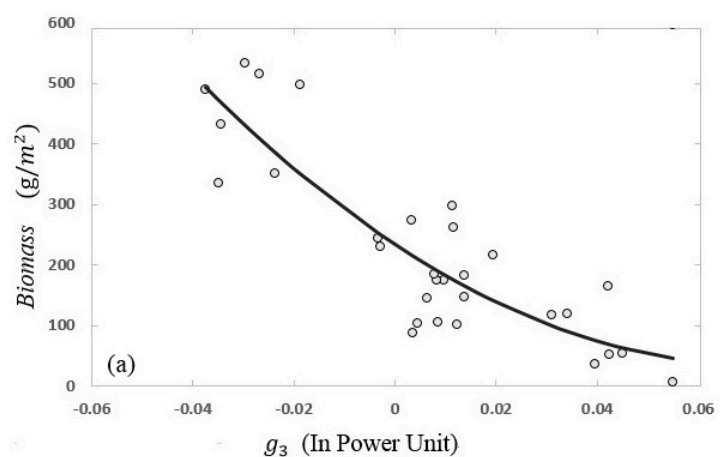

Biomass $=234.43-5504.11 \times g_{3}+37944.54 \times g_{3}^{2}$ $R^{2}=0.765 R M S E=73.2$
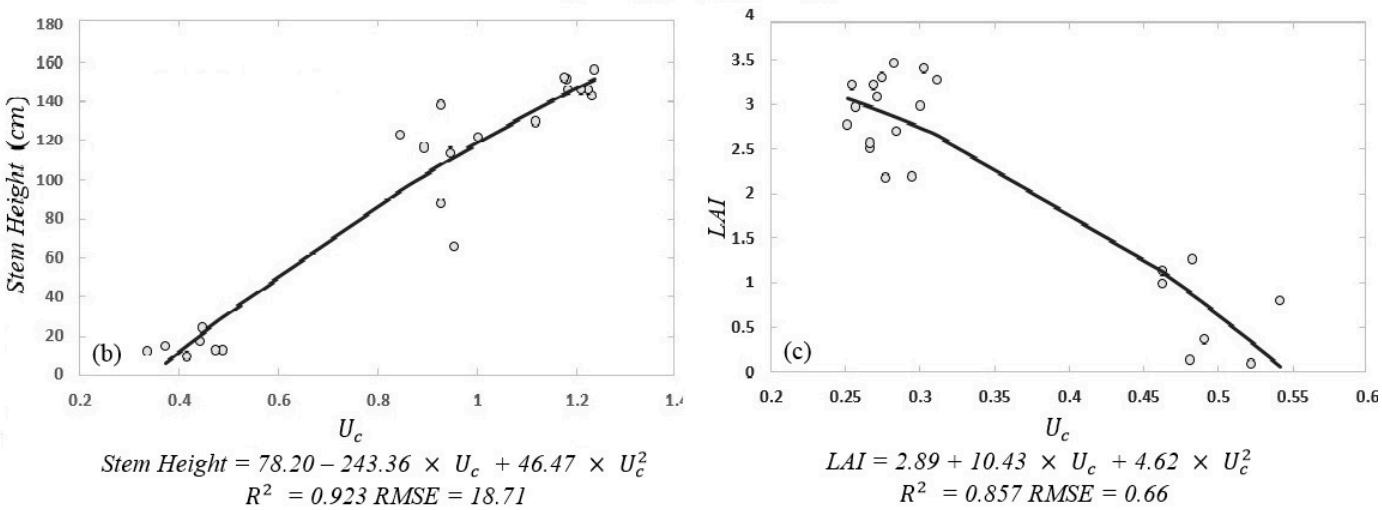

Figure 10. The best single regression models for rape biomass, stem height and LAI inversion. (a) The best single parameter regression model for biomass; (b) The best single parameter regression model for stem height; (c) The best single parameter regression model for LAI.

\section{Discussion}

In this study, four groups' $\mathrm{CP}$ observable behaviors were investigated as a function of DAS during the entire growth cycle of rape. With the comparison of the evolution of biomass, stem height and LAI, some $\mathrm{CP}$ observables provided obvious physical potential for growth parameter inversion; at the same time, they also showed high importance in three growth parameters' inversion with the RF algorithm. However, other parameters that showed a nonlinear relationship with growth parameters and were difficult for physical mechanism interpretation also played an important role in growth parameters inversion with RF algorithm. Anyway, RF showed good performance for biomass, stem height and LAI inversion with CP parameters than traditional single parameter regression empirical models. The parameters of Stokes child parameters showed the best performance for the rape scattering mechanism interpretation compared to the other groups. The performance of Stokes parameters in the interpretation of rape growth also showed better results according to the RF importance analysis. Decomposition and the backscattering coefficient are also useful ways for crop mapping, monitoring and biomass inversion in previous literature [3,21,23,27]. Among these studies, [21] focuses on crops mapping; [27] was limited in polarimetric decomposition of full polarization; [3] and [23] were limited to the small scale of $\mathrm{CP}$ parameters and focused on rice crops. In our case, CP decomposition parameters, backscattering coefficients and Stokes-related parameters were extracted and analyzed.

The decomposition components in our case showed ambiguity for scattering mechanism analysis because of the over-estimation of volume components. However, double-bounce scattering showed perfect coincidence with three growth parameter at P2, P3 and P4, which agrees with previous literature [27]. Backscatter coefficient results agreed with the literature [23].

The results of this study revealed a high sensitivity of different $\mathrm{CP}$ parameters to rape growth during all growing stages, and an effective and robust method for rape growth parameters inversion. 
Our estimation results were encouraging when compared to those reported by other studies. For example, Yang [25], due to only using a simple empirical linear model and a single CP parameter, obtained RMSE for biomass inversion of $56.5 \mathrm{~g} / \mathrm{m}^{2}$ and $\mathrm{R}^{2}$ of 0.92 . More recently, an RMSE of $32.3 \mathrm{~g} / \mathrm{m}^{2}$ was obtained for wheat biomass estimation with the RF algorithm by Li in [4]. However, these results were obtained using HJ-CCD satellite data, which are not available in most regions. Taking the full potential for exploring CP observables in rape growth parameter inversion and the effectiveness and robustness of the inversion model, our research seemed to be more promising for operational applications by farm condition and practice. Nevertheless, due to the long revisit cycle of Radarsat-2 (24 day), as well as the different sowing dates, resulting in crop growth differences among different rape fields, which led to an ambiguous response of $\mathrm{P} 3$, which includes three different $\mathrm{BBCH}$ scales, we should refine the inversion model by short revisit time satellites when available in the future.

The potential capability of CP SAR observables for rape growth parameters inversion was confirmed by this study; the robustness and high accuracy of the RF algorithm in biomass, stem height and LAI inversion were also revealed in this study. It also confirmed that CP parameters are able to monitor the dynamic change of rape growth parameters during the main growth cycle. The RF algorithm is efficient for a large parameter dataset and not sensitive to noise or over-fitting in parameter inversion. Despite that the experiment was conducted in a cold temperate area and that rape types and cultivation practices differ in an important way all over the Earth, the main findings of the work could be extrapolated to other sites, since they are based on the scattering responses that are common for rape during their growth cycle.

\section{Conclusions}

This paper described CP response on rape growth parameters like biomass, stem height and LAI and their inversion with these CP parameters by the RF algorithm. The study was based on four groups, 27 CP parameters. Five hundred decision tree were used in RF for three growth parameters' inversion. At each node per decision tree, nine CP parameters were randomly chosen for inversion rules.

The study included two research aspects. First, CP SAR data response of rape was analyzed during the entire rape growth cycle. Based on this, the sensitivity of $\mathrm{CP}$ observables for biomass, stem height and LAI was described. Meanwhile, the scattering mechanism of rape at different stages of the growth cycle and the reason for its induction were analyzed. Second, the growth parameters including biomass, stem height and LAI were inversed with the RF algorithm. With the importance analysis of $27 \mathrm{CP}$ observables, 33 samples were chosen for biomass model building and 11 for estimated result accuracy validation, 22 samples for stem height and LAI model building and 11 for validation. The importance analysis results, which include both parameters with a similar trend to the evolution of growth parameters and parameters with no obvious coincidence trend with growth parameters, showed the valuable ability of the RF algorithm in complex, nonlinear system model building. The high determination coefficient $\left(\mathrm{R}^{2}\right)$ and low RMSE between the estimation results and measured values also confirmed the robustness of the RF algorithm in crop growth parameters inversion. Among all of these CP parameters, $g_{0}$ and $g_{3}$ in the Stokes group, $\mathrm{m}, \delta, \chi, P_{c}$ and $U_{c}$ in the Stokes child group, $\sigma_{\mathrm{RH}}, \sigma_{\mathrm{RR}}$ and the ratio of $\sigma_{\mathrm{RL}}$ and $\sigma_{\mathrm{RR}}$ in the backscattering coefficients and the volume scattering component in $\mathrm{CP}$ decomposition group had higher sensitivity to the growth parameters. Therefore, these $\mathrm{CP}$ parameters were suggested for growth parameters inversion with regression models.

Although this work has focused on the oilseed rape crop of hybrid-5, the general approach may be applicable to other species and other crops. However, since this study was performed on a per-field basis, ambiguity could be produced by the heterogeneity inside the field if the growth parameters need to be estimated by a pixel-wise scale. If they do, the uncertainty should be identified.

Moreover, despite the effectiveness of the RF method for rape growth parameters inversion, it is difficult to relate parameters' importance to the physical scattering mechanism of crops. In contrast, the parameters applied in the regression models make sense for the physical mechanism interpretation. 
In the future, finding an eclectic method between these two methods for growth parameters inversion will be studied.

Acknowledgments: This work was supported in part by the National Basic Research Program of China (Grant No. 2013CB733404), the project of The Technique of Accurate Surface Parameters Inversion Using GF-3 Images (Grant No. 03-Y20A11-9001-15/16) and National Natural Science Foundation of China (41401477). The authors would also like to thank the four anonymous referees who have helped improve the original manuscript.

Author Contributions: Wangfei Zhang is the principal author of this manuscript having written the majority of the manuscript and contributed during one phase of the investigation. The other co-authors contributed to the field logistics, the experiment performance, the selection and interpretation of the methods and some portions of the written manuscript. The order of the authors reflects their level of contribution.

Conflicts of Interest: The authors declare no conflict of interest.

\section{References}

1. Liu, C. Development of Rapeseed Production and Bio-Diesel in China; Huazhong Agricultural University: Wuhan, China, 2008.

2. Liu, J.; Pattey, E.; Jégo, G. Assessment of vegetation indices for regional crop green LAI estimation from Landsat images over multiple growing seasons. Remote Sens. Environ. 2012, 123, 347-358. [CrossRef]

3. Lopez-Sanchez, J.M.; Vicente-Guijalba, F.; Ballester-Berman, J.D.; Cloude, S.R. Polarimetric Response of Rice Fields at C-Band: Analysis and Phenology Retrieval. IEEE Trans. Geosci. Remote Sens. 2014, 52, 2977-2993. [CrossRef]

4. Wang, L.A.; Zhou, W.; Zhu, X.K.; Dong, Z.D.; Guo, W.S. Estimation of biomass in wheat using random forest regression algorithm and remote sensing data. Crop J. 2016, 4, 212-219. [CrossRef]

5. Jia, Y.Z.; Li, B.; Cheng, Y.Z.; Liu, T.; Guo, Y.; Wu, X.H.; Wang, L.G. Comparison between GF-1 images and Landsat-8 images in monitoring maize LAI. Trans. Chin. Soc. Agric. Eng. 2015, 31, 173-179.

6. Skriver, H. Crop Classification by Multitemporal C- and L-Band Single- and Dual-Polarization and Fully Polarimetric SAR. IEEE Trans. Geosci. Remote Sens. 2012, 50, 2138-2149. [CrossRef]

7. Küçük, Ç.; Taşkın, G.; Erten, E. Paddy-Rice Phenology Classification Based on Machine-Learning Methods Using Multitemporal Co-Polar X-Band SAR Images. IEEE. Sel. Top. Appl. Earth Obs. Remote Sens. 2016, 9, 2509-2519. [CrossRef]

8. Hoang, H.K.; Bernier, M.; Duchesne, S.; Tran, Y.M. Rice Mapping Using RADARSAT-2 Dual- and Quad-Pol Data in a Complex Land-Use Watershed: Cau River Basin (Vietnam). IEEE J. Sel. Top. Appl. Earth Obs. Remote Sens. 2016, 9, 3082-3096. [CrossRef]

9. Lopez-Sanchez, J.M.; Cloude, S.R.; Ballester-Berman, J.D. Rice Phenology Monitoring by Means of SAR Polarimetry at X-Band. IEEE Trans. Geosci. Remote Sens. 2012, 50, 2695-2709. [CrossRef]

10. Jin, X.; Yang, G.; Xu, X.; Yang, H.; Feng, H. Combined Multi-Temporal Optical and Radar Parameters for Estimating LAI and Biomass in Winter Wheat Using HJ and RADARSAR-2 Data. Remote Sens. 2015, 7, 13251-13272. [CrossRef]

11. Jia, M.; Tong, W.; Zhang, Y.; Chen, Y. Rice Biomass Estimation Using Radar Backscattering Data at S-band. IEEE J. Sel. Top. Appl. Earth Obs. Remote Sens. 2014, 7, 469-479. [CrossRef]

12. Alberti, G.; Candido, P.; Peressotti, A.; Turco, S.; Piussi, P.; Zerbi, G. Abstract and Résumé. Aboveground biomass relationships for mixed ash (L. and Hudson) stands in Eastern Prealps of Friuli Venezia Giulia (Italy). Ann. For. Sci. 2005, 62, 831-836. [CrossRef]

13. Cloude, S. Polarisation: Applications in Remote Sensing, 1st ed.; Oxford University Press: Oxford, UK, 2009.

14. Rui, Y.K.; Peng, Y.F.; Wang, Z.R.; Shen, J.B. Stem perimeter, height and biomass of maize (Zea mays L.) grown under different $\mathrm{N}$ fertilization regimes in Beijing, China. Int. J. Plant Prod. 2009, 3, 85-90.

15. Shang, F.; Hirose, A. Averaged Stokes Vector Based Polarimetric SAR Data Interpretation. IEEE Trans. Geosci. Remote Sens. 2015, 53, 4536-4547. [CrossRef]

16. Cloude, S.R.; Pottier, E. A review of target decomposition theorems in radar polarimetry. IEEE Trans. Geosci. Remote Sens. 1996, 34, 498-518. [CrossRef]

17. Raney, R.K. Hybrid-Polarity SAR Architecture. IEEE Trans. Geosci. Remote Sens. 2007, 45, 3397-3404. [CrossRef] 
18. Raney, R.K. Dual-polarized SAR and Stokes parameters. IEEE Geosci. Remote Sens. Lett. 2006, 3, 317-319. [CrossRef]

19. Steele-Dunne, S.C.; McNairn, H.; Monsivais-Huertero, A. Radar Remote Sensing of Agricultural Canopies: A Review. IEEE J. Sel. Top. Appl. Earth Obs. Remote Sens. 2017, 10, 2249-2273. [CrossRef]

20. Mcnairn, H.; Shang, J.; Champagne, C.; Jiao, X. TerraSAR-X and RADARSAT-2 for crop classification and acreage estimation. In Proceedings of the 2009 IEEE International Geoscience and Remote Sensing Symposium, Cape Town, South Africa, 12-17 July 2009.

21. Charbonneau, F.J.; Brisco, B.; Raney, R.K.; McNairn, H.; Liu, C.; Vachon, P.W.; Shang, J.; DeAbreu, R.; Chanmpagne, C.; Merzouki, A.; et al. Compact polarimetry overview and applications assessment. Can. J. Remote Sens. 2014, 36, 298. [CrossRef]

22. Nord, M.E.; Ainsworth, T.L.; Lee, J.S.; Stacy, N.J.S. Comparison of Compact Polarimetric Synthetic Aperture Radar Modes. IEEE Trans. Geosci. Remote Sens. 2009, 47, 174-188. [CrossRef]

23. Yang, Z.; Li, K.; Liu, L.; Shao, Y.; Brisco, B.; Li, W.G. Rice growth monitoring using simulated compact polarimetric C band SAR. Radio Sci. 2014, 49, 1300-1315. [CrossRef]

24. Cloude, S.R.; Goodenough, D.G.; Chen, H. Compact Decomposition Theory. Geosci. Remote Sens. Lett. IEEE 2012, 9, 28-32. [CrossRef]

25. Yang, H.; Xie, L.; Chen, E.X.; Zhang, H.; Yang, G.J.; Li, Z.H.; Gu, X.H. Biomass estimation of oilseed rape using simulated compact polarimtric SAR imagery. In Proceedings of the 2016 IEEE International Geoscience and Remote Sensing Symposium (IGARSS), Beijing, China, 10-15 July 2016.

26. Tottman, D.R. The decimal code for the growth stages of cereals, with illustrations. Ann. Appl. Biol. 2008, 110, 441-454. [CrossRef]

27. Yang, H.; Li, Z.Y.; Chen, E.X.; Zhao, C.J.; Yang, G.J.; Casa, R.; Pignatti, S.; Feng, Q. Temporal Polarimetric Behavior of Oilseed Rape (Brassica napus L.) at C-Band for Early Season Sowing Date Monitoring. Remote Sens. 2014, 6, 10375-10394. [CrossRef]

28. Raney, R.K.; Cahill, J.S.T.S.; Patterson, G.W.; Bussey, D.B.J. The m-chi decomposition of hybrid dual-polarimetric radar data. In Proceedings of the 2012 IEEE International Geoscience and Remote Sensing Symposium, Munich, Germany, 22-27 July 2012.

29. Stokes, G.G. On the Composition and Resolution of Streams of Polarized Light from different Sources. Trans. Camb. Philos. Soc. 1851, 9, 399.

30. Zhang, H.; Xie, L.; Wang, C.; Wu, F.; Zhang, B. Investigation of the Capability of H- $\alpha$ Decomposition of Compact Polarimetric SAR. IEEE Geosci. Remote Sens. Lett. 2014, 11, 868-872. [CrossRef]

31. Breiman, L. Random Forests. Mach. Learn. 2001, 45, 5-32. [CrossRef]

32. Cutler, A.; Cutler, D.R.; Stevens, J.R. Random Forests. Mach. Learn. 2012, 45, 157-176.

33. Bouvet, A.; Toan, T.L.; Lam-Dao, N. Monitoring of the Rice Cropping System in the Mekong Delta Using ENVISAT/ASAR Dual Polarization Data. IEEE Trans. Geosci. Remote Sens. 2009, 47, 517-526. [CrossRef]

34. Larranaga, A.; Alvarez-Mozos, J.; Albizua, L.; Peters, J. Backscattering Behavior of Rain-Fed Crops Along the Growing Season. Geosci. Remote Sens. Lett. IEEE 2013, 10, 386-390. [CrossRef]

35. Born, M.; Wolf, E. Principles of Optics, 6th ed.; Cambridge University Press: Cambridge, UK, 1999.

(c) 2017 by the authors. Licensee MDPI, Basel, Switzerland. This article is an open access article distributed under the terms and conditions of the Creative Commons Attribution (CC BY) license (http://creativecommons.org/licenses/by/4.0/). 